\title{
Improved robustness for nearly-incompressible large deformation meshfree simulations on Delaunay tessellations
}

\author{
A. Ortiz-Bernardin ${ }^{\mathrm{a}, *}$, M. A. Puso ${ }^{\mathrm{b}}$, N. Sukumar ${ }^{\mathrm{c}}$ \\ ${ }^{a}$ Department of Mechanical Engineering, University of Chile, Av. Beauchef 851, Santiago \\ 8370456, Chile. \\ ${ }^{b}$ Lawrence Livermore National Laboratory, P.O. Box 808, Livermore, CA 94551, U.S.A. \\ ${ }^{c}$ Department of Civil and Environmental Engineering, University of California, Davis, CA 95616, \\ USA.
}

\section{Abstract}

A displacement-based Galerkin meshfree method for large deformation analysis of nearly-incompressible elastic solids is presented. Nodal discretization of the domain is defined by a Delaunay tessellation (three-node triangles and four-node tetrahedra), which is used to form the meshfree basis functions and to numerically integrate the weak form integrals. In the proposed approach for nearly-incompressible solids, a volume-averaged nodal projection operator is constructed to average the dilatational constraint at a node from the displacement field of surrounding nodes. The nodal dilatational constraint is then projected onto the linear approximation space. The displacement field is constructed on the linear space and enriched with bubble-like meshfree basis functions for stability. The new procedure leads to a displacementbased formulation that is similar to $F$-bar methodologies in finite elements and isogeometric analysis. We adopt maximum-entropy meshfree basis functions, and the performance of the meshfree method is demonstrated on benchmark problems using structured and unstructured background meshes in two and three dimensions.

\footnotetext{
*Corresponding author. Tel: +56 (2) 297846 64, Fax: +56 (2) 268960 57,

Email address: aortizb@ing.uchile.cl (A. Ortiz-Bernardin)
}

Preprint submitted to Comp. Meth. Appl. Mech. Engr.

May 7, 2015 
The nonlinear simulations reveal that the proposed methodology provides improved robustness for nearly-incompressible large deformation analysis on Delaunay meshes. Keywords: hyperelasticity, large deformations, meshfree methods, maximum-entropy approximation, $F$-bar method, Delaunay meshes

\section{Introduction}

In nearly-incompressible analysis of solids that undergo large deformations, mesh distortion introduces a limitation for practical use of simplicial (Delaunay) tessellations within the framework of standard finite elements. Three-node triangular and four-node tetrahedral finite elements are not used for nearly-incompressible analysis of solids because they lead to volumetric locking. However, they can be suitably modified for nearly-incompressible settings through the displacement/pressure mixed formulation ( $u-p$ form). The realization of these finite elements is the well-known MINI element [1], where the nodes located at the vertices of the simplicial element are used to interpolate continuous linear displacement and continuous linear pressure fields. In addition, the displacement field is enriched with an interior node located at the barycenter of the simplicial element. This extra node is related to a cubic (bubble) basis function that vanishes on the element boundary and renders an inf-sup stable element [2-4]. Although the MINI element demonstrated better stability properties than several finite element formulations in certain finite deformation regimes [5], the shape functions dependence on the Delaunay tessellation makes it very sensitive to mesh distortion. In this paper, a new methodology on Delaunay meshes is proposed for the meshfree analysis of nearly-incompressible solids at finite strains that is superior to the MINI element formulation.

In the literature, the poor performance of simplicial tessellations in large deformation analysis of nearly-incompressible solids has been improved through various 
techniques such as mixed-enhanced elements [6-8], pressure stabilization [9-11], composite pressure fields [12-14], and average nodal pressure/strains [15-20]. The last two approaches are broadly based on the idea of reducing pressure (dilatational) constraints to alleviate volumetric locking. In meshfree methods, nodal integration techniques [21] can be considered to be indirectly related to methods that use simplicial tessellations since their formulation is based on the dual of the Delaunay triangulation, that is, the Voronoi diagram. In this approach, the fewer constraints that are met by performing numerical integration only at the nodes permits to alleviate volumetric locking. However, the drawback of nodal integration techniques is their instability, which has motivated studies to stabilize them [22, 23].

In contrast to finite elements, meshfree methods are constructed using basis functions that possess larger supports and do not rely on a mesh for their definition. This allows meshfree methods some degree of insensitivity to mesh distortions, thus providing us with the motivation to use meshfree basis functions in this paper. Nonetheless, a background mesh is still required in Galerkin meshfree methods to perform the numerical integration of the weak form integrals. In the meshfree method that is developed herein, background meshes of three-node triangles in two dimensions and four-node tetrahedra in three dimensions, are used.

Volumetric locking remains an issue in meshfree methods that use simplicial tessellations for numerical integration in nearly-incompressible media problems. Thus, a special procedure needs to be developed to alleviate volumetric locking. To this end the nonlinear version of the volume-averaged nodal projection method (referred to as VANP in Ref. [24]) proposed for small strain elasticity in Ref. [25] is developed to average the dilatational constraint at a node from the displacement field of surrounding nodes. The nodal dilatational constraint is then projected onto the linear approximation space. The displacement field is constructed on the linear space and 
enriched with bubble-like meshfree basis functions for stability. The formulation so devised leads to a displacement-based method that shares some common features with the F-bar-Patch method of Ref. [26] and the isogeometric F-bar projection method of Ref. [27], and as such, it can be regarded as an F-bar methodology for meshfree methods. In the numerical implementation, maximum-entropy basis functions are used as the meshfree basis functions. Another approach that uses bubble functions to address volumetric locking for low-order simplicial tessellations is proposed in Ref. [28] for compressible and nearly-incompressible linear elastic solids and in Refs. [29, 30] for large deformations. Wu and Koishi [30] use the conforming nodal integration procedure of Chen et al. [31] to suppress locking, whereas in our approach, the locking-free behavior stems from a $u-p$ mixed formulation in which a volume-averaged technique is used to eliminate the pressure degrees of freedom from the analysis. Furthermore, the smoothing in Ref. [30] is done over the covering that is formed by the bubble nodes that are neighbors to an element face and the nodes that define that face, whereas in this work the volume-averaging is done over the region of support of the vertex basis functions.

In a Galerkin-based meshfree method, the integration domain is a cell that typically does not coincide with the region that is defined by the intersecting supports of two overlapping meshfree basis functions. In addition, meshfree basis functions are rational (nonpolynomial) functions. These are two central issues that introduce numerical errors when using standard Gauss quadrature for numerical integration. The errors can be reduced by using a large number of Gauss points per cell; however, this substantially increases the computational costs in the numerical integration. There have been many attempts to correct these integration errors. An early contribution was due to Dolbow and Belytschko [32], who proposed to use integration cells that were aligned with the support of the nodal basis functions. Since then, many other 
approaches have been pursued (for instance, see Refs. [33, 34]). Babuška and coworkers have provided the theoretical basis for the numerical integration issue in first-order meshfree methods [35] as well as higher-order meshfree approximations [36]. Other approaches that are based on nodal integration ideas [31] construct a strain correction that significantly reduces integration errors. Ortiz et al. [25] proposed a strain correction based on a smoothing procedure for linear approximations on triangular and quadrilateral background meshes and extended these ideas to tetrahedral background meshes in Ref. [37]. Duan et al. [38] proposed a smoothing procedure for second-order approximations on triangular background meshes. Chen et al. [39] proposed a variationally consistent integration method for high-order meshfree approximations that generalizes the notion of nodal integration and is applicable for Gauss quadrature on triangles and squares. Recently, Duan et al. [40] used the Hu-Washizu three-field variational principle to demonstrate the variational consistency of the second-order accurate integration scheme previously presented in Ref. [38] for meshfree methods on triangular meshes and an extension of this scheme to third-order accuracy was also provided. The corresponding second-order accurate integration scheme for four-node tetrahedral meshes is presented in Duan et al. [41].

The nonlinear weak form integrals in the VANP method are more involved and therefore more accurate integration schemes are required. One of the findings in the development of the work herein was that the strain correction procedure proposed earlier by the authors $[25,37]$ was not effective for integration of the nonlinear weak VANP form. Although this correction exactly satisfied linear patch tests and was second-order accurate, it was found to be insufficiently robust for large strains. The integration by Duan et al. [38, 41] on the other hand is second-order accurate and satisfies the quadratic patch test and thus offers more regularity, which provides better robustness in two- and three-dimensional computations. 
The main contributions in this paper for the analysis of nearly-incompressible solids at finite strains are as follows:

- A robust displacement-based formulation is developed for Delaunay tessellations via modifications to the standard $u-p$ mixed weak form. To the best of our knowledge, this approach has not previously appeared in the literature.

- The use of bubble-like meshfree basis functions in our formulation provides the necessary stability of the pressure field. As a consequence, it delivers smooth pressure fields on Delaunay tessellations. This feature is not present in other formulations for low-order tessellations.

- The integration method of Duan et al. [41], which proved better than the integration method developed in our earlier papers [25, 37], is implemented for the first time in 3D large deformations.

- In comparing the proposed formulation with its closest finite element counterpart, the MINI element, it allows larger deformations and delivers more accurate solutions with smoother pressure fields.

The remainder of the paper is organized as follows. Section 2 presents a summary of maximum-entropy basis functions. The formulation proposed for nearlyincompressible elastic solids at finite strains is developed in Section 3. Here, the volume-averaged nodal projection (VANP) method is developed via modifications to the standard u-p mixed weak form. The discrete equations are provided in Section 4, and the numerical integration scheme used in the meshfree method is outlined in Section 5. Numerical examples are presented in Section 6 to demonstrate the performance of the meshfree method in large deformation analysis of nearly-incompressible elastic solids. Some concluding remarks are given in Section 7. 


\section{Maximum-entropy basis functions}

Meshfree basis functions typically do not vanish on the domain boundary, which precludes direct imposition of essential boundary conditions; hence procedures such as Lagrange multipliers, penalty methods or Nitsche's method among others must be used to enforce essential boundary conditions (for details, see Ref. [42]). However, maximum-entropy (max-ent) basis functions [43-45] vanish on the boundary of a convex domain [44], which allows direct imposition of essential boundary conditions at the nodes. Because of this feature, max-ent basis functions are selected in the VANP formulation.

Consider a convex domain represented by a set of $n$ scattered nodes and a prior (weight) function $w_{a}(\boldsymbol{X})$ associated with node $a$. On using the Shannon-Jaynes entropy functional [45], the set of max-ent basis functions $\left\{\phi_{a}(\boldsymbol{X}) \geq 0\right\}_{a=1}^{n}$ that define the approximation function $\boldsymbol{u}_{h}(\boldsymbol{X})=\sum_{a} \phi_{a}(\boldsymbol{X}) \boldsymbol{u}_{a}\left(\boldsymbol{u}_{a}\right.$ are nodal coefficients), is obtained via the solution of the following concave optimization problem:

$$
\max _{\phi \in \mathbb{R}_{+}^{n}}-\sum_{a=1}^{n} \phi_{a}(\boldsymbol{X}) \ln \left(\frac{\phi_{a}(\boldsymbol{X})}{w_{a}(\boldsymbol{X})}\right)
$$

subject to the linear reproducing conditions:

$$
\sum_{a=1}^{n} \phi_{a}(\boldsymbol{X})=1 \quad \sum_{a=1}^{n} \phi_{a}(\boldsymbol{X}) \boldsymbol{c}_{a}=\mathbf{0}
$$

where $\boldsymbol{c}_{a}=\boldsymbol{X}_{a}-\boldsymbol{X}$ are shifted nodal coordinates and $\mathbb{R}_{+}^{n}$ is the non-negative orthant. Typical priors that can be used include kernel or window functions that are well-known in the meshfree literature. In this paper, we use a $C^{2}$ quartic polynomial given by

$$
w_{a}(q)=\left\{\begin{array}{lr}
1-6 q^{2}+8 q^{3}-3 q^{4} & 0 \leq q \leq 1 \\
0 & q>1
\end{array}\right.
$$


where $q=\left\|\boldsymbol{X}_{a}-\boldsymbol{X}\right\| / \rho_{a}$ and $\rho_{a}=\gamma h_{a}$ is the support radius of the basis function of node $a ; \gamma$ is a parameter that controls the support-width of the basis function, and $h_{a}$ is a characteristic nodal spacing associated with node $a$.

On using Lagrange multipliers, the solution of the variational problem (1) is [45]:

$$
\phi_{a}(\boldsymbol{X})=\frac{Z_{a}\left(\boldsymbol{X} ; \boldsymbol{\lambda}^{*}\right)}{Z\left(\boldsymbol{X} ; \boldsymbol{\lambda}^{*}\right)}, \quad Z_{a}\left(\boldsymbol{X} ; \boldsymbol{\lambda}^{*}\right)=w_{a}(\boldsymbol{X}) \exp \left(-\boldsymbol{\lambda}^{*} \cdot \boldsymbol{c}_{a}\right),
$$

where $Z\left(\boldsymbol{X} ; \boldsymbol{\lambda}^{*}\right)=\sum_{b} Z_{b}\left(\boldsymbol{X} ; \boldsymbol{\lambda}^{*}\right)$ and $\boldsymbol{\lambda}^{*}=\left[\lambda_{1}^{*} \lambda_{2}^{*} \lambda_{3}^{*}\right]^{\mathrm{T}}$ in three dimensions. In (3), the Lagrange multiplier vector $\boldsymbol{\lambda}^{*}$ is the minimizer of the dual optimization problem:

$$
\boldsymbol{\lambda}^{*}=\arg \min _{\boldsymbol{\lambda} \in \mathbb{R}^{d}} \ln Z(\boldsymbol{X} ; \boldsymbol{\lambda})
$$

which leads to a system of $d$ nonlinear equations:

$$
F(\boldsymbol{\lambda})=\nabla_{\boldsymbol{\lambda}} \ln Z(\boldsymbol{\lambda})=-\sum_{a}^{n} \phi_{a}(\boldsymbol{X}) \boldsymbol{c}_{a}=\mathbf{0}
$$

where $d$ is the spatial dimension and $\nabla_{\boldsymbol{\lambda}}$ refers to the gradient with respect to $\boldsymbol{\lambda}$. Once the converged $\boldsymbol{\lambda}^{*}$ is found, the basis functions are computed from (3) and the gradient of the basis functions is [46]:

$\nabla \phi_{a}=\phi_{a}\left\{\boldsymbol{c}_{a} \cdot\left[(\mathrm{H})^{-1}-(\mathrm{H})^{-1} \cdot \mathrm{A}\right]-\sum_{b=1}^{n} \frac{\nabla w_{b} \exp \left(-\boldsymbol{\lambda}^{*} \cdot \boldsymbol{c}_{b}\right)}{Z}\right\}+\frac{\nabla w_{a} \exp \left(-\boldsymbol{\lambda}^{*} \cdot \boldsymbol{c}_{a}\right)}{Z}$

where

$$
\mathrm{A}=\sum_{b=1}^{n} \boldsymbol{c}_{b} \otimes \frac{\nabla w_{b} \exp \left(-\boldsymbol{\lambda}^{*} \cdot \boldsymbol{c}_{b}\right)}{Z}
$$

and $\mathrm{H}$ is the Hessian matrix defined by

$$
\mathrm{H}=\nabla_{\boldsymbol{\lambda}} F=\nabla_{\boldsymbol{\lambda}} \nabla_{\boldsymbol{\lambda}} \ln Z=\sum_{b=1}^{n} \phi_{b} \boldsymbol{c}_{b} \otimes \boldsymbol{c}_{b}
$$

with $\otimes$ denoting the dyadic product. 


\section{Variational formulation}

Consider an elastic body with open domain $\Omega \subset \mathbb{R}^{d}(d=2,3)$ in the initial (reference) configuration. The domain is bounded by the $(d-1)$-dimensional surface $\partial \Omega$ whose unit outward normal is $\boldsymbol{N}$. A mapping, denoted as $\chi$, defines the displacement $\boldsymbol{u}$ of a particle from its initial position $\boldsymbol{X}$ to its current position $\boldsymbol{x}$, that is, $\boldsymbol{u}=\chi(\boldsymbol{X})-\boldsymbol{X}=\boldsymbol{x}-\boldsymbol{X}$. The boundary is assumed to admit the decompositions $\partial \Omega=\partial \Omega_{\chi} \cup \partial \Omega_{t}$ and $\emptyset=\partial \Omega_{\chi} \cap \partial \Omega_{t}$, where $\partial \Omega_{\chi}$ is the portion of the boundary where the deformation $\chi=\hat{\chi}$ is prescribed and $\partial \Omega_{t}$ is the portion of the boundary where the external surface forces $\hat{\boldsymbol{t}}_{0}$ (assumed to be independent of the motion) are applied. The deformations are required to be admissible, which means they belong to the space:

$$
\mathscr{D}=\left\{\chi: \Omega \rightarrow \mathbb{R}^{d} \mid \operatorname{det} \boldsymbol{F}>0, \chi=\hat{\chi} \text { on } \partial \Omega_{\chi}\right\}
$$

where

$$
\boldsymbol{F}=\nabla_{0} \chi=\nabla_{0} \boldsymbol{X}=\boldsymbol{I}+\nabla_{0} \boldsymbol{u}
$$

is the deformation gradient tensor ${ }^{1} ; \boldsymbol{I}$ is the identity tensor.

The kinematic relation between the Green-Lagrange strain tensor $\boldsymbol{E}(\chi)$ and the deformation gradient tensor $\boldsymbol{F}(\chi)$ is:

$$
\boldsymbol{E}=\frac{1}{2}\left(\boldsymbol{F}^{\mathrm{T}} \boldsymbol{F}-\boldsymbol{I}\right)
$$

which can be expressed in terms of the right Cauchy-Green deformation tensor $\boldsymbol{C}=$ $\boldsymbol{F}^{\mathrm{T}} \boldsymbol{F}$.

The elastic body is assumed to be homogeneous and isotropic. The second Piola-

\footnotetext{
${ }^{1}$ Subscript 0 is used to refer to operations in the initial configuration.
} 
Kirchhoff stress $\boldsymbol{S}(\chi)$ is related nonlinearly to the strain $\boldsymbol{E}(\chi)$ by

$$
\boldsymbol{S}=\frac{\partial \Psi(\boldsymbol{E}(\chi))}{\partial \boldsymbol{E}}
$$

where $\Psi$ is a strain energy function, which in addition to $\boldsymbol{E}$, depends on Lamé's first $(\lambda)$ and second $(\mu)$ material parameters.

\subsection{Displacement-based weak form}

The potential energy functional for an elastic body that is subjected to external surface forces and is free of body forces is given as

$$
\Pi(\chi)=\int_{\Omega} \Psi(\boldsymbol{E}(\chi)) d V-\int_{\partial \Omega_{t}} \hat{\boldsymbol{t}}_{0} \cdot \chi d S
$$

The stationarity of (11) in the arbitrary direction $\boldsymbol{v} \in \mathscr{V}=\left\{\boldsymbol{v}: \Omega \rightarrow \mathbb{R}^{d} \mid \boldsymbol{v}=\right.$ $\mathbf{0}$ on $\left.\partial \Omega_{\chi}\right\}$ is given by the directional derivative

$$
\left.\mathrm{D} \Pi(\chi)[\boldsymbol{v}] \equiv \frac{d \Pi(\chi+\epsilon \boldsymbol{v})}{d \epsilon}\right|_{\epsilon=0}=0
$$

and yields the displacement-based weak form as follows:

$$
\mathrm{D} \Pi(\chi)[\boldsymbol{v}]=\int_{\Omega} \boldsymbol{S}: \mathrm{D} \boldsymbol{E}(\chi)[\boldsymbol{v}] d V-\int_{\partial \Omega_{t}} \hat{\boldsymbol{t}}_{0} \cdot \boldsymbol{v} d S=0
$$

where $\mathrm{D} \boldsymbol{E}(\chi)[\boldsymbol{v}]$ can be proved (for instance, see Ref. [47]) to be

$$
\mathrm{D} \boldsymbol{E}(\chi)[\boldsymbol{v}]=\left(\boldsymbol{F}^{\mathrm{T}} \boldsymbol{\nabla}_{0} \boldsymbol{v}\right)_{\mathrm{sym}}
$$

On substituting (14) into (13) leads to the final displacement-based weak form expression as

$$
\mathrm{D} \Pi(\chi)[\boldsymbol{v}]=\int_{\Omega} \boldsymbol{S}:\left(\boldsymbol{F}^{\mathrm{T}} \boldsymbol{\nabla}_{0} \boldsymbol{v}\right)_{\mathrm{sym}} d V-\int_{\partial \Omega_{t}} \hat{\boldsymbol{t}}_{0} \cdot \boldsymbol{v} d S=0 .
$$

A nearly-incompressible material must satisfy the limit $J=\operatorname{det} \boldsymbol{F} \rightarrow 1$, which is achieved by setting the Lamé parameters such that the Poisson's ratio approaches 
$1 / 2$. However, imposing this severe constraint on the kinematic behavior leads to volumetric locking when using the displacement-based weak form (15) [48]. This indicates that the weak form (15) cannot be used to describe movement while simultaneously satisfying $J \approx 1$. Volumetric locking manifests itself in numerical formulations and can be suppressed using the $u-p$ mixed formulation.

\section{2. $u$-p mixed weak form}

The $u-p$ mixed formulation is a two-field variational problem in which, in addition to the displacement field, a second independent variable is chosen to represent the hydrostatic pressure field. In order to identify the hydrostatic pressure variable in the formulation, the strain energy function is decomposed into its deviatoric and volumetric parts:

$$
\Psi(J, \boldsymbol{E})=\Psi^{\mathrm{dev}}(J, \boldsymbol{E})+\Psi^{\mathrm{dil}}(J),
$$

which redefines the potential energy functional as follows:

$$
\Pi(\chi)=\int_{\Omega} \Psi^{\mathrm{dev}}(J, \boldsymbol{E}(\chi)) d V+\int_{\Omega} \Psi^{\mathrm{dil}}(J) d V-\int_{\partial \Omega_{t}} \hat{\boldsymbol{t}}_{0} \cdot \chi d S .
$$

As in (11), the stationarity of (17) is obtained by applying the directional derivative, which leads to the weak form

$$
\mathrm{D} \Pi(\chi)[\boldsymbol{v}]=\int_{\Omega}\left(\boldsymbol{S}^{\mathrm{dev}}+\boldsymbol{S}^{\mathrm{dil}}\right):\left(\boldsymbol{F}^{\mathrm{T}} \boldsymbol{\nabla}_{0} \boldsymbol{v}\right)_{\mathrm{sym}} d V-\int_{\partial \Omega_{t}} \hat{\boldsymbol{t}}_{0} \cdot \boldsymbol{v} d S=0,
$$

where

$$
\boldsymbol{S}^{\mathrm{dev}}=\frac{\partial \Psi^{\mathrm{dev}}}{\partial \boldsymbol{E}}
$$

and

$$
\boldsymbol{S}^{\mathrm{dil}}=\frac{d \Psi^{\mathrm{dil}}}{d J} \frac{\partial J}{\partial \boldsymbol{E}}=p J \boldsymbol{C}^{-1} .
$$

In (20), the hydrostatic pressure $p=\frac{d \Psi^{\text {dil }}}{d J}$ and the identity $\frac{\partial J}{\partial \boldsymbol{E}}=J \boldsymbol{C}^{-1}$ have been used (for instance, see Ref. [47]). 
On defining the pressure space as $\mathscr{P}:=\left\{p: p \in L^{2}(\Omega), \int_{\Omega} p d \Omega=0\right\}$ and introducing $p \in \mathscr{P}$ as an independent pressure trial function with $q \in \mathscr{P}$ an arbitrary pressure test function, yields the following $u-p$ mixed variational form:

$$
\begin{gathered}
\int_{\Omega}\left(\boldsymbol{S}^{\mathrm{dev}}+p J \boldsymbol{C}^{-1}\right):\left(\boldsymbol{F}^{\mathrm{T}} \boldsymbol{\nabla}_{0} \boldsymbol{v}\right)_{\mathrm{sym}} d V-\int_{\partial \Omega_{t}} \hat{\boldsymbol{t}}_{0} \cdot \boldsymbol{v} d S=0 \\
\int_{\Omega} q\left(p-\frac{d \Psi^{\mathrm{dil}}}{d J}\right) d V=0
\end{gathered}
$$

\subsection{Volume-averaged nodal projection method}

An alternative locking-free approach, where the only variable is the displacement field, can be developed starting from the multiplicative decomposition of the deformation gradient tensor into its deviatoric and dilatational parts: $\boldsymbol{F}=\boldsymbol{F}^{\text {dil }} \boldsymbol{F}^{\text {dev }}[49]$, and using this to define a modified deformation gradient tensor as follows [50]:

$$
\overline{\boldsymbol{F}}=\overline{\boldsymbol{F}}^{\mathrm{dil}} \boldsymbol{F}^{\mathrm{dev}}
$$

where $\overline{\boldsymbol{F}}^{\text {dil }}=\bar{J}^{1 / 3} \boldsymbol{I}$ and $\boldsymbol{F}^{\text {dev }}=J^{-1 / 3} \boldsymbol{F}$, which leads to

$$
\operatorname{det} \overline{\boldsymbol{F}}=\bar{J}
$$

since $\operatorname{det} \boldsymbol{F}^{\text {dev }}=1$. In $(22), \overline{\boldsymbol{F}}^{\text {dil }}$ is a modified tensor that suppresses volumetric locking [27]. For convenience in further derivations, (22) is rewritten as

$$
\overline{\boldsymbol{F}}=\alpha \boldsymbol{F},
$$

where $\alpha=(\bar{J} / J)^{1 / 3}$. An identity akin to $(24)$ is the basis for the $F$-bar methodology used in finite elements $[12,50]$ and isogeometric analysis [27].

From (24) it is clear that the key ingredient in the modified displacement-based weak form for meshfree methods proposed herein is to find an appropriate definition for $\bar{J}$ such that volumetric locking is suppressed. In other words, an explicit definition 
for the 'bar' operator needs to be developed. In brief, the idea is to find such an operator from the pressure constraint (21b) of the $u-p$ mixed weak form. Before proceeding, special tessellations will be defined for constructing $\bar{J}$ and for numerical integration in the meshfree method.

Let the domain tessellation with simplices be denoted by $\mathcal{T}(\Omega)$. The tessellation consists of three-node triangular or four-node tetrahedral cells denoted by $\mathcal{C}$. The vertices of the tessellation are used to define the standard node set $\mathcal{N}^{s}$. In addition to the standard node set, we define a barycenter node set as $\mathcal{N}^{b}$ with nodes located at the barycenter of each cell $\mathcal{C}$ in the tessellation $\mathcal{T}(\Omega)$. So, an enhanced node set is defined as $\mathcal{N}^{+}=\mathcal{N}^{s} \cup \mathcal{N}^{b}$. Fig. 1 depicts a schematic representation of a two-dimensional simplicial tessellation with its corresponding cells and node set definitions. In our approach, the simplicial tessellation $\mathcal{T}(\Omega)$ that connects the standard node set $\mathcal{N}^{s}$ is generated using a meshing software and the Gauss points locations for the numerical integration procedure are computed based on this mesh. The enhanced node set $\mathcal{N}^{+}$ is constructed when needed by including the additional nodes that are required in the standard node set.

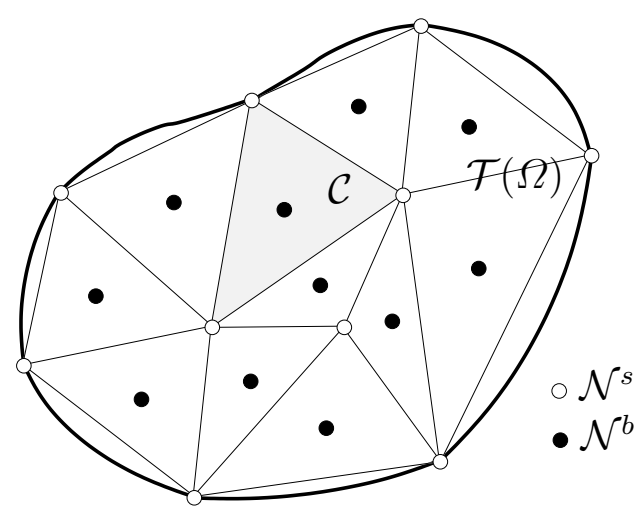

Fig. 1: Schematic representation of a two-dimensional simplicial tessellation $\mathcal{T}(\Omega)$ for the enhanced node set $\mathcal{N}^{+}=\mathcal{N}^{s} \cup \mathcal{N}^{b}$. The shaded area is a representative cell denoted by $\mathcal{C}$. 
The starting step for developing the bar operator is the discretization of the pressure constraint (21b) using

$$
\begin{aligned}
& p_{h}(\boldsymbol{X})=\sum_{b=1}^{n} \phi_{b}(\boldsymbol{X}) p_{b}, \\
& q_{h}(\boldsymbol{X})=\sum_{c=1}^{n} \phi_{c}(\boldsymbol{X}) q_{c}
\end{aligned}
$$

where $n$ is the number of nodes in the node set $\mathcal{N}^{s}$, whose associated meshfree basis functions $\phi_{i}(i=b, c)$ have a nonzero discrete value at the sampling point $\boldsymbol{X}$. On substituting (25) into the pressure constraint (21b) and relying on the arbitrariness of nodal pressure test functions yields

$$
\sum_{b=1}^{n_{b}} \int_{\Omega} \phi_{c}(\boldsymbol{X}) \phi_{b}(\boldsymbol{X}) p_{b} d V-\int_{\Omega} \phi_{c}(\boldsymbol{X}) \frac{d \Psi^{\mathrm{dil}}}{d J} d V=0
$$

and performing row-sum on the discrete pressure term leads to

$$
\left\{\int_{\Omega} \phi_{c}(\boldsymbol{X}) d V\right\} p_{c}-\int_{\Omega} \phi_{c}(\boldsymbol{X}) \frac{d \Psi^{\text {dil }}}{d J} d V=0
$$

Finally, solving for $p_{c}$ in (27) gives the following volume-averaged nodal pressure:

$$
p_{c}=\frac{\int_{\Omega_{c}} \phi_{c}(\boldsymbol{X}) \frac{d \Psi^{\mathrm{dil}}}{d J} d V}{\int_{\Omega_{c}} \phi_{c}(\boldsymbol{X}) d V}
$$

where the integration volume $\Omega$ has been replaced with $\Omega_{c}$, the union of cells that are attached to node $c$ (see Fig. 2). Equation (28) is the nonlinear version of the volume-averaged nodal pressure used in Refs. [24, 25] for linear elasticity.

To realize the bar operator, as an example we consider the following dilatational strain energy:

$$
\Psi^{\text {dil }}=\frac{1}{2} \kappa(J-1)^{2}
$$


where $\kappa=\lambda+\frac{2}{3} \mu$ is the bulk modulus of the material. Thus, $\frac{d \Psi^{\mathrm{dil}}}{d J}=\kappa(J-1)$ and (28) yields

$$
p_{c}=\kappa \frac{\int_{\Omega_{c}} \phi_{c}(\boldsymbol{X}) J d V}{\int_{\Omega_{c}} \phi_{c}(\boldsymbol{X}) d V}-\kappa=\kappa\left(J_{c}-1\right)=\kappa\left(\pi_{c}[J]-1\right)
$$

where the volume-averaged nodal projection (VANP) operator $\pi_{c}$ is:

$$
\pi_{c}[\cdot]=\frac{\int_{\Omega_{c}} \phi_{c}(\boldsymbol{X})[\cdot] d V}{\int_{\Omega_{c}} \phi_{c}(\boldsymbol{X}) d V}
$$

The adoption of the name 'projection' reflects the fact that (28) stems from the pressure constraint $(21 \mathrm{~b})$, which is like an $L^{2}$ projection. Note the similarity of the operator (31) with the assumed gradient operator of Ref. [20] (see (16) therein) and the assumed strain nodal matrix of Ref. [51] (see (18) therein).

As can be inferred from (30), the nodal operator applied to $J$ gives its nodal representation as

$$
J_{c}=\pi_{c}[J]=\frac{\int_{\Omega_{c}} \phi_{c}(\boldsymbol{X})[J] d V}{\int_{\Omega_{c}} \phi_{c}(\boldsymbol{X}) d V} .
$$

Finally, by the linear combination $p_{h}=\sum_{c} \phi_{c} p_{c}$, the bar operator is given by the projection operator as

$$
\pi[\cdot]=\sum_{c=1}^{n_{c}} \phi_{c}(\boldsymbol{X}) \pi_{c}[\cdot]=\sum_{c=1}^{n_{c}} \phi_{c}(\boldsymbol{X})\left\{\frac{\int_{\Omega_{c}} \phi_{c}(\boldsymbol{X})[\cdot] d V}{\int_{\Omega_{c}} \phi_{c}(\boldsymbol{X}) d V}\right\}
$$

Thus, $\bar{J}$ is computed as follows:

$$
\bar{J}=\pi[J]=\sum_{c=1}^{n_{c}} \phi_{c}(\boldsymbol{X}) \pi_{c}[J]=\sum_{c=1}^{n_{c}} \phi_{c}(\boldsymbol{X})\left\{\frac{\int_{\Omega_{c}} \phi_{c}(\boldsymbol{X}) J d V}{\int_{\Omega_{c}} \phi_{c}(\boldsymbol{X}) d V}\right\}=\sum_{c=1}^{n_{c}} \phi_{c}(\boldsymbol{X}) J_{c}
$$

As shown above, the VANP approach is based on modifications to the standard $u-p$ mixed formulation. Consequently, some consideration to the inf-sup condition [24] should follow. In lieu of an analytical treatment, the proposed formulation here 


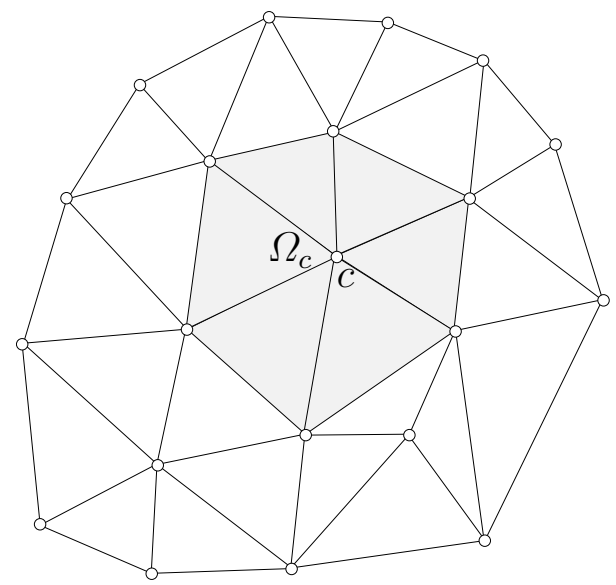

Fig. 2: Schematic representation of the standard node set $\mathcal{N}^{s}$. The shaded region illustrates an integration volume for the computation of the VANP operator associated with node $c$.

is designed to be very close in form to the inf-sup stable MINI element, i.e., the displacement field is enhanced with additional nodes located at the barycenter of the simplices and these nodes carry displacement degrees of freedom. In the VANP formulation, max-ent basis functions are used to approximate the displacement and pressure fields. However, no pressure degrees of freedom are associated with the barycentric nodes, and the constraint ratio for the VANP approach is the same as the MINI element. We point out that the max-ent basis function that is associated with the barycentric node does not vanish on the boundary of the corresponding simplex. However, as the support gets tighter for the max-ent basis function that is associated with the barycentric node, the basis function tends to a bubble function on the simplex. Hence, we refer to these barycenter nodes as bubble-like nodes. So, following the preceding considerations, first-order max-ent basis functions are used to construct the space $\mathscr{P}_{h}\left(\mathscr{U}_{h}\right)$ with the standard node set $\mathcal{N}^{s}$ and the space $\mathscr{U}_{h}$ with the enhanced node set $\mathcal{N}^{+}$. For implementation purposes of the VANP operator, this means the basis functions that appear in the operator (33) are computed using 
the standard node set $\mathcal{N}^{s}$, but $J=\operatorname{det} \boldsymbol{F}$ in (34) is computed using the enhanced node set $\mathcal{N}^{+}$since $\boldsymbol{F}$ is computed from the displacement field.

To achieve a symmetric tangent stiffness matrix, $\overline{\boldsymbol{F}}$ is introduced in the energy function, which leads to the following modified potential energy functional:

$$
\bar{\Pi}(\chi)=\int_{\Omega} \Psi(\overline{\boldsymbol{E}}(\chi)) d V-\int_{\partial \Omega_{t}} \hat{\boldsymbol{t}}_{0} \cdot \chi d S,
$$

where $\Psi(\overline{\mathbf{E}}(\chi))$ is the modified strain energy function, which depends on $\overline{\boldsymbol{F}}$ through the modified Green-Lagrange strain tensor:

$$
\overline{\boldsymbol{E}}=\frac{1}{2}\left(\overline{\boldsymbol{F}}^{\mathrm{T}} \overline{\boldsymbol{F}}-\boldsymbol{I}\right) .
$$

The locking-free modified displacement-based weak form is obtained by taking the directional derivative in (35), which gives

$$
\mathrm{D} \bar{\Pi}(\chi)[\boldsymbol{v}]=\int_{\Omega} \overline{\boldsymbol{S}}: \mathrm{D} \overline{\boldsymbol{E}}(\chi)[\boldsymbol{v}] d V-\int_{\partial \Omega_{t}} \hat{\boldsymbol{t}}_{0} \cdot \boldsymbol{v} d S=0 .
$$

In (37), the modified second Piola-Kirchhoff stress tensor $\overline{\boldsymbol{S}}(\chi)$ is defined as

$$
\overline{\boldsymbol{S}}=\boldsymbol{S}(\overline{\boldsymbol{E}}(\chi))=\frac{\partial \Psi(\boldsymbol{E})}{\partial \boldsymbol{E}}(\overline{\boldsymbol{E}}(\chi))
$$

and $\mathrm{D} \overline{\boldsymbol{E}}(\chi)[\boldsymbol{v}]$ needs to be developed. To this end, the following operator is defined:

$$
\theta[\cdot]=\frac{\pi[\cdot]}{\pi[J]}-\frac{1}{J}\{\cdot\} .
$$

The complete derivation of $\mathrm{D} \overline{\boldsymbol{E}}(\chi)[\boldsymbol{v}]$ is presented in Appendix A. The final expression is

$$
\mathrm{D} \overline{\boldsymbol{E}}(\chi)[\boldsymbol{v}]=\frac{1}{3} \theta\left[J \operatorname{tr}\left(\boldsymbol{\nabla}_{0} \boldsymbol{v} \boldsymbol{F}^{-1}\right)\right] \overline{\boldsymbol{F}}^{\mathrm{T}} \overline{\boldsymbol{F}}+\alpha\left(\overline{\boldsymbol{F}}^{\mathrm{T}} \boldsymbol{\nabla}_{0} \boldsymbol{v}\right)_{\mathrm{sym}} .
$$

On substituting (40) into (37) leads to the final modified displacement-based weak form expression:

$$
\begin{aligned}
\mathrm{D} \bar{\Pi}(\chi)[\boldsymbol{v}]= & \frac{1}{3} \int_{\Omega} \theta\left[J \operatorname{tr}\left(\boldsymbol{\nabla}_{0} \boldsymbol{v} \boldsymbol{F}^{-1}\right)\right] \overline{\boldsymbol{S}}:\left(\overline{\boldsymbol{F}}^{\mathrm{T}} \overline{\boldsymbol{F}}\right) d V+\int_{\Omega} \alpha \overline{\boldsymbol{S}}:\left(\overline{\boldsymbol{F}}^{\mathrm{T}} \boldsymbol{\nabla}_{0} \boldsymbol{v}\right)_{\mathrm{sym}} d V \\
& -\int_{\partial \Omega_{t}} \hat{\boldsymbol{t}}_{0} \cdot \boldsymbol{v} d S=0 .
\end{aligned}
$$




\subsection{Linearization}

The implicit numerical solution relies on the linearization of the weak form (41). The linearized weak form in the direction of the increment $\Delta \boldsymbol{u}$ is given by

$$
\mathrm{D} \bar{\Pi}(\chi)[\boldsymbol{v}]+\mathrm{D}^{2} \bar{\Pi}(\chi)[\boldsymbol{v}, \Delta \boldsymbol{u}]=0
$$

where $\mathrm{D}^{2} \bar{\Pi}(\chi)[\boldsymbol{v}, \Delta \boldsymbol{u}] \equiv \mathrm{D}\{\mathrm{D} \bar{\Pi}(\chi)[\boldsymbol{v}]\}[\Delta \boldsymbol{u}]$ is the second variation of $\bar{\Pi}(\chi)$ along $\Delta \boldsymbol{u}$. The complete derivation of the second variation is given in Appendix B. Only the final result is presented here. The external forces are assumed to be independent of the motion, and for the sake of clarity, the second variation is split into material and geometric parts:

$$
\mathrm{D}^{2} \bar{\Pi}(\chi)[\boldsymbol{v}, \Delta \boldsymbol{u}]=\left(\mathrm{D}^{2} \bar{\Pi}(\chi)[\boldsymbol{v}, \Delta \boldsymbol{u}]\right)_{\mathrm{mat}}+\left(\mathrm{D}^{2} \bar{\Pi}(\chi)[\boldsymbol{v}, \Delta \boldsymbol{u}]\right)_{\mathrm{geo}}
$$

where

$$
\begin{aligned}
\left(\mathrm{D}^{2} \bar{\Pi}(\chi)[\boldsymbol{v}, \Delta \boldsymbol{u}]\right)_{\mathrm{mat}}= & +\frac{1}{9} \int_{\Omega} \theta\left[J \operatorname{tr}\left(\boldsymbol{\nabla}_{0} \boldsymbol{v} \boldsymbol{F}^{-1}\right)\right] \theta\left[J \operatorname{tr}\left(\boldsymbol{\nabla}_{0} \Delta \boldsymbol{u} \boldsymbol{F}^{-1}\right)\right]\left(\overline{\boldsymbol{F}}^{\mathrm{T}} \overline{\boldsymbol{F}}\right): \overline{\mathcal{D}}:\left(\overline{\boldsymbol{F}}^{\mathrm{T}} \overline{\boldsymbol{F}}\right) d V \\
& +\frac{1}{3} \int_{\Omega} \alpha \theta\left[J \operatorname{tr}\left(\boldsymbol{\nabla}_{0} \boldsymbol{v} \boldsymbol{F}^{-1}\right)\right]\left(\overline{\boldsymbol{F}}^{\mathrm{T}} \overline{\boldsymbol{F}}\right): \overline{\mathcal{D}}:\left(\overline{\boldsymbol{F}}^{\mathrm{T}} \boldsymbol{\nabla}_{0} \Delta \boldsymbol{u}\right)_{\mathrm{sym}} d V \\
& +\frac{1}{3} \int_{\Omega} \alpha\left(\overline{\boldsymbol{F}}^{\mathrm{T}} \boldsymbol{\nabla}_{0} \boldsymbol{v}\right)_{\mathrm{sym}}: \overline{\mathcal{D}}:\left(\overline{\boldsymbol{F}}^{\mathrm{T}} \overline{\boldsymbol{F}}\right) \theta\left[J \operatorname{tr}\left(\boldsymbol{\nabla}_{0} \Delta \boldsymbol{u} \boldsymbol{F}^{-1}\right)\right] d V \\
& +\int_{\Omega} \alpha^{2}\left(\overline{\boldsymbol{F}}^{\mathrm{T}} \boldsymbol{\nabla}_{0} \boldsymbol{v}\right)_{\mathrm{sym}}: \overline{\mathcal{D}}:\left(\overline{\boldsymbol{F}}^{\mathrm{T}} \boldsymbol{\nabla}_{0} \Delta \boldsymbol{u}\right)_{\mathrm{sym}} d V
\end{aligned}
$$


and

$$
\begin{aligned}
\left(\mathrm{D}^{2} \bar{\Pi}(\chi)[\boldsymbol{v}, \Delta \boldsymbol{u}]\right)_{\mathrm{geo}}= & -\frac{1}{3} \int_{\Omega} \frac{1}{\bar{J}^{2}} \pi\left[J \operatorname{tr}\left(\boldsymbol{\nabla}_{0} \boldsymbol{v} \boldsymbol{F}^{-1}\right)\right] \pi\left[J \operatorname{tr}\left(\boldsymbol{\nabla}_{0} \Delta \boldsymbol{u} \boldsymbol{F}^{-1}\right)\right] \overline{\boldsymbol{S}}:\left(\overline{\boldsymbol{F}}^{\mathrm{T}} \overline{\boldsymbol{F}}\right) d V \\
& +\frac{1}{3} \int_{\Omega} \frac{1}{\bar{J}} \pi\left[J \operatorname{tr}\left(\boldsymbol{\nabla}_{0} \boldsymbol{v} \boldsymbol{F}^{-1}\right) \operatorname{tr}\left(\boldsymbol{\nabla}_{0} \Delta \boldsymbol{u} \boldsymbol{F}^{-1}\right)\right] \overline{\boldsymbol{S}}:\left(\overline{\boldsymbol{F}}^{\mathrm{T}} \overline{\boldsymbol{F}}\right) d V \\
& -\frac{1}{3} \int_{\Omega} \frac{1}{\bar{J}} \pi\left[J \operatorname{tr}\left(\boldsymbol{\nabla}_{0} \boldsymbol{v} \boldsymbol{F}^{-1} \boldsymbol{\nabla}_{0} \Delta \boldsymbol{u} \boldsymbol{F}^{-1}\right)\right] \overline{\boldsymbol{S}}:\left(\overline{\boldsymbol{F}}^{\mathrm{T}} \overline{\boldsymbol{F}}\right) d V \\
& +\frac{1}{3} \int_{\Omega} \operatorname{tr}\left(\boldsymbol{\nabla}_{0} \boldsymbol{v} \boldsymbol{F}^{-1} \boldsymbol{\nabla}_{0} \Delta \boldsymbol{u} \boldsymbol{F}^{-1}\right) \overline{\boldsymbol{S}}:\left(\overline{\boldsymbol{F}}^{\mathrm{T}} \overline{\boldsymbol{F}}\right) d V \\
& +\frac{2}{9} \int_{\Omega} \theta\left[J \operatorname{tr}\left(\boldsymbol{\nabla}_{0} \boldsymbol{v} \boldsymbol{F}^{-1}\right)\right] \theta\left[J \operatorname{tr}\left(\boldsymbol{\nabla}_{0} \Delta \boldsymbol{u} \boldsymbol{F}^{-1}\right)\right] \overline{\boldsymbol{S}}:(\overline{\boldsymbol{F}} \overline{\mathrm{F}}) d V \\
& +\frac{2}{3} \int_{\Omega} \alpha \theta\left[J \operatorname{tr}\left(\boldsymbol{\nabla}_{0} \boldsymbol{v} \boldsymbol{F}^{-1}\right)\right] \overline{\boldsymbol{S}}:\left(\overline{\boldsymbol{F}}^{\mathrm{T}} \boldsymbol{\nabla}_{0} \Delta \boldsymbol{u}\right)_{\mathrm{sym}} d V \\
& +\frac{2}{3} \int_{\Omega} \alpha\left(\overline{\boldsymbol{F}}^{\mathrm{T}} \boldsymbol{\nabla}_{0} \boldsymbol{v}\right)_{\mathrm{sym}}: \overline{\boldsymbol{S}} \theta\left[J \operatorname{tr}\left(\boldsymbol{\nabla}_{0} \Delta \boldsymbol{u} \boldsymbol{F}^{-1}\right)\right] d V \\
& +\int_{\Omega} \alpha^{2} \overline{\boldsymbol{S}}:\left[\left(\boldsymbol{\nabla}_{0} \boldsymbol{v}\right)^{\mathrm{T}} \boldsymbol{\nabla}_{0} \Delta \boldsymbol{u}\right]_{\mathrm{sym}} d V .
\end{aligned}
$$

In (43b), the modified Lagrangian elasticity tensor $\overline{\mathcal{D}}$ is computed as follows:

$$
\overline{\mathcal{D}}=\mathcal{D}(\overline{\boldsymbol{E}}(\chi))=\frac{\partial \boldsymbol{S}(\boldsymbol{E})}{\partial \boldsymbol{E}}(\overline{\boldsymbol{E}}(\chi))
$$

Finally, upon substituting (41) and (43) into (42) yields the final expression for the linearized weak form.

\section{Discrete equations}

The discretization of the linearized weak form (42) leads to the following NewtonRaphson scheme:

$$
{ }^{t+\Delta t}\left(\mathrm{~K}_{\mathrm{mat}}+\mathrm{K}_{\mathrm{geo}}\right){ }^{(i-1)} \Delta \mathrm{u}^{(i)}={ }^{t+\Delta t} \mathrm{~F}-{ }^{t+\Delta t} \mathrm{~T}^{(i-1)}={ }^{t+\Delta t} \mathrm{R}^{(i-1)},
$$

where $\mathrm{K}_{\text {mat }}$ and $\mathrm{K}_{\text {geo }}$ are the material and geometric global tangent stiffness matrices, respectively; $\mathrm{F}$ and $\mathrm{T}$ are the external and internal global nodal force column vectors, 
respectively; $\mathrm{R}$ is the residual global nodal force column vector; and $\Delta \mathrm{u}$ is the column vector that collects all the displacement degrees of freedom of the mesh. On the other hand, $t+\Delta t$ denotes the incremental approach where a solution is known at a discrete time $t$ and the solution at a discrete time $t+\Delta t$ is sought; the increment $\Delta t$ corresponds to the load step or load increment. Finally, $i$ stands for the equilibrium iterations within an increment. The global tangent stiffness matrices as well as the global nodal force column vectors are obtained by assembling the nodal contributions.

The discrete material and geometric tangent stiffness matrices, as well as the nodal force column vectors, are derived using the maximum-entropy approximation for both the test and trial functions in the reference configuration, as follows:

$$
\begin{aligned}
\Delta \boldsymbol{u}_{h}(\boldsymbol{X}) & =\sum_{p=1}^{n} \phi_{p}(\boldsymbol{X}) \Delta \boldsymbol{u}_{p} \\
\boldsymbol{v}_{h}(\boldsymbol{X}) & =\sum_{q=1}^{n} \phi_{q}(\boldsymbol{X}) \boldsymbol{v}_{q} .
\end{aligned}
$$

The basis functions $\phi_{i}(i=p, q)$ are computed using the nodal information of the node set $\mathcal{N}^{+}$of the background mesh. The same mesh is used to locate the Gauss points for numerical integration of the linearized weak form integrals. Thus, the integration domain becomes the elements of the background mesh and is denoted as $\Omega^{e}$. However, we recall that the projection operator (or equivalently, the bar operator) is computed using the standard node set $\mathcal{N}^{s}$ (see Section 3.3 for details).

To simplify the exposition, the explicit expressions for the tangent stiffness matrices and residual nodal force vector are provided only in three-dimensions. To this end, the following matrix notations, which result from symmetry conditions, are used:

$$
\begin{aligned}
& \overline{\mathrm{C}}=\left\{\overline{\boldsymbol{F}}^{\mathrm{T}} \overline{\boldsymbol{F}}\right\}=\left[\begin{array}{llllll}
\bar{C}_{11} & \bar{C}_{22} & \bar{C}_{33} & 2 \bar{C}_{12} & 2 \bar{C}_{13} & 2 \bar{C}_{23}
\end{array}\right]^{\mathrm{T}}, \\
& \overline{\mathrm{S}}=\{\overline{\boldsymbol{S}}\}=\left[\begin{array}{llllll}
\bar{S}_{11} & \bar{S}_{22} & \bar{S}_{33} & \bar{S}_{12} & \bar{S}_{13} & \bar{S}_{23}
\end{array}\right]^{\mathrm{T}},
\end{aligned}
$$




$$
\overline{\mathrm{D}}=\{\overline{\mathcal{D}}\}=\left[\begin{array}{cccccc}
\overline{\mathcal{D}}_{1111} & \overline{\mathcal{D}}_{1122} & \overline{\mathcal{D}}_{1133} & \overline{\mathcal{D}}_{1112} & \overline{\mathcal{D}}_{1113} & \overline{\mathcal{D}}_{1123} \\
& \overline{\mathcal{D}}_{2222} & \overline{\mathcal{D}}_{2233} & \overline{\mathcal{D}}_{2212} & \overline{\mathcal{D}}_{2213} & \overline{\mathcal{D}}_{2223} \\
& & \overline{\mathcal{D}}_{3333} & \overline{\mathcal{D}}_{3312} & \overline{\mathcal{D}}_{3313} & \overline{\mathcal{D}}_{3323} \\
& & & \overline{\mathcal{D}}_{1212} & \overline{\mathcal{D}}_{1213} & \overline{\mathcal{D}}_{1223} \\
& & & & \overline{\mathcal{D}}_{1313} & \overline{\mathcal{D}}_{1323} \\
& \text { sym. } & & & & \overline{\mathcal{D}}_{2323}
\end{array}\right],
$$

where $\{\cdot\}$ denotes Voigt notation. The following matrix forms, which result from the discretization procedure, are also used:

$$
\begin{aligned}
& \mathrm{B}_{q}=\left[\begin{array}{ccc}
\left(F_{11}\right)^{-1} \phi_{q, X} & 0 & 0 \\
0 & \left(F_{22}\right)^{-1} \phi_{q, Y} & 0 \\
0 & 0 & \left(F_{33}\right)^{-1} \phi_{q, Z} \\
\left(F_{21}\right)^{-1} \phi_{q, Y} & \left(F_{12}\right)^{-1} \phi_{q, X} & 0 \\
\left(F_{31}\right)^{-1} \phi_{q, Z} & 0 & \left(F_{13}\right)^{-1} \phi_{q, X} \\
0 & \left(F_{32}\right)^{-1} \phi_{q, Z} & \left(F_{23}\right)^{-1} \phi_{q, Y}
\end{array}\right] \\
& \overline{\mathrm{B}}_{q}^{0}=\left[\begin{array}{ccc}
\bar{F}_{11} \phi_{q, X} & \bar{F}_{21} \phi_{q, X} & \bar{F}_{31} \phi_{q, X} \\
\bar{F}_{12} \phi_{q, Y} & \bar{F}_{22} \phi_{q, Y} & \bar{F}_{32} \phi_{q, Y} \\
\bar{F}_{13} \phi_{q, Z} & \bar{F}_{23} \phi_{q, Z} & \bar{F}_{33} \phi_{q, Z} \\
\bar{F}_{12} \phi_{q, X}+\bar{F}_{11} \phi_{q, Y} & \bar{F}_{22} \phi_{q, X}+\bar{F}_{21} \phi_{q, Y} & \bar{F}_{32} \phi_{q, X}+\bar{F}_{31} \phi_{q, Y} \\
\bar{F}_{13} \phi_{q, X}+\bar{F}_{11} \phi_{q, Z} & \bar{F}_{23} \phi_{q, X}+\bar{F}_{21} \phi_{q, Z} & \bar{F}_{33} \phi_{q, X}+\bar{F}_{31} \phi_{q, Z} \\
\bar{F}_{13} \phi_{q, Y}+\bar{F}_{12} \phi_{q, Z} & \bar{F}_{23} \phi_{q, Y}+\bar{F}_{22} \phi_{q, Z} & \bar{F}_{33} \phi_{q, Y}+\bar{F}_{32} \phi_{q, Z}
\end{array}\right],
\end{aligned}
$$




$$
\overline{\mathrm{B}}_{q}^{k k}=\left[\begin{array}{c}
\theta\left[J \mathrm{~m}^{\mathrm{T}} \mathrm{B}_{q}\right] \bar{C}_{11} \\
\theta\left[J \mathrm{~m}^{\mathrm{T}} \mathrm{B}_{q}\right] \bar{C}_{22} \\
\theta\left[J \mathrm{~m}^{\mathrm{T}} \mathrm{B}_{q}\right] \bar{C}_{33} \\
2 \theta\left[J \mathrm{~m}^{\mathrm{T}} \mathrm{B}_{q}\right] \bar{C}_{12} \\
2 \theta\left[J \mathrm{~m}^{\mathrm{T}} \mathrm{B}_{q}\right] \bar{C}_{13} \\
2 \theta\left[J \mathrm{~m}^{\mathrm{T}} \mathrm{B}_{q}\right] \bar{C}_{23}
\end{array}\right],
$$

Additionally, the following vector, which arises from some trace operations, is defined:

$$
\mathrm{m}=\left[\begin{array}{llllll}
1 & 1 & 1 & 1 & 1 & 1
\end{array}\right]^{\mathrm{T}}
$$

\subsection{Residual nodal force vector}

The residual nodal force vector is obtained upon discretization of (41). This gives

$$
\mathrm{R}_{q}=\int_{\partial \Omega_{t}^{e}} \phi_{q} \hat{\boldsymbol{t}}_{0} d S-\frac{1}{3} \int_{\Omega^{e}} \theta\left[J \mathrm{~B}_{q}^{\mathrm{T}} \mathrm{m}\right] \overline{\mathrm{D}}^{\mathrm{T}} \overline{\mathrm{S}} d V-\int_{\Omega^{e}} \alpha\left(\overline{\mathrm{B}}_{q}^{0}\right)^{\mathrm{T}} \overline{\mathrm{S}} d V .
$$

\subsection{Material tangent stiffness matrix}

The material tangent stiffness matrix is obtained upon discretization of (43b). Before proceeding, the terms in (43b) are rearranged such that the following expression is obtained:

$$
\left(\mathrm{D}^{2} \bar{\Pi}(\chi)[\boldsymbol{v}, \Delta \boldsymbol{u}]\right)_{\mathrm{mat}}=\int_{\Omega} \overline{\boldsymbol{\nabla}_{0} \boldsymbol{v}}: \overline{\mathcal{D}}: \overline{\nabla_{0} \Delta \boldsymbol{u}} d V
$$

where

$$
\overline{\nabla_{0}(\cdot)}=\frac{1}{3} \theta\left[J \operatorname{tr}\left(\nabla_{0}(\cdot) \boldsymbol{F}^{-1}\right)\right] \overline{\boldsymbol{C}}+\alpha\left(\overline{\boldsymbol{F}}^{\mathrm{T}} \boldsymbol{\nabla}_{0}(\cdot)\right)_{\mathrm{sym}} .
$$

Due to the symmetry of (56), further simplifications can be done using Voigt notation to obtain

$$
\left(\mathrm{D}^{2} \bar{\Pi}(\chi)[\boldsymbol{v}, \Delta \boldsymbol{u}]\right)_{\mathrm{mat}}=\int_{\Omega}\left\{\overline{\boldsymbol{\nabla}_{0} \boldsymbol{v}}\right\}^{\mathrm{T}}\{\overline{\mathcal{D}}\}\left\{\overline{\boldsymbol{\nabla}_{0} \Delta \boldsymbol{u}}\right\} d V
$$


Finally, on substituting (46) into (58) leads to the following discrete material tangent stiffness matrix:

$$
\left(\mathrm{K}_{\text {mat }}\right)_{p q}=\int_{\Omega^{e}} \overline{\mathrm{B}}_{p}^{\mathrm{T}} \overline{\mathrm{D}} \overline{\mathrm{B}}_{q} d V
$$

\subsection{Geometric tangent stiffness matrix}

On substituting (46) into (43c) yields the following discrete geometric tangent stiffness matrix:

$$
\begin{aligned}
\left(\mathrm{K}_{\text {geo }}\right)_{p q}= & -\frac{1}{3} \int_{\Omega^{e}} \frac{1}{\bar{J}^{2}} \pi\left[J \mathrm{~B}_{p}^{\mathrm{T}} \mathrm{m}\right] \pi\left[J \mathrm{~m}^{\mathrm{T}} \mathrm{B}_{q}\right] \overline{\mathrm{C}}^{\mathrm{T}} \overline{\mathrm{S}} d V \\
& +\frac{1}{3} \int_{\Omega^{e}} \frac{1}{\bar{J}} \pi\left[J \mathrm{~B}_{p}^{\mathrm{T}} \mathrm{mm}^{\mathrm{T}} \mathrm{B}_{q}\right] \overline{\mathrm{C}}^{\mathrm{T}} \overline{\mathrm{S}} d V \\
& -\frac{1}{3} \int_{\Omega^{e}} \frac{1}{\bar{J}} \pi\left[J \boldsymbol{F}^{-\mathrm{T}}\left(\boldsymbol{\nabla}_{0} \phi_{p}\right)\left(\boldsymbol{\nabla}_{0} \phi_{q}\right)^{\mathrm{T}} \boldsymbol{F}^{-1}\right] \overline{\mathrm{C}}^{\mathrm{T}} \overline{\mathrm{S}} d V \\
& +\frac{1}{3} \int_{\Omega^{e}} \boldsymbol{F}^{-\mathrm{T}}\left(\boldsymbol{\nabla}_{0} \phi_{p}\right)\left(\boldsymbol{\nabla}_{0} \phi_{q}\right)^{\mathrm{T}} \boldsymbol{F}^{-1} \overline{\mathrm{C}}^{\mathrm{T}} \overline{\mathrm{S}} d V \\
& +\frac{2}{9} \int_{\Omega^{e}} \theta\left[J \mathrm{~B}_{p}^{\mathrm{T}} \mathrm{m}\right] \theta\left[J \mathrm{~m}^{\mathrm{T}} \mathrm{B}_{q}\right] \overline{\mathrm{C}}^{\mathrm{T}} \overline{\mathrm{S}} d V \\
& +\frac{2}{3} \int_{\Omega^{e}} \alpha \theta\left[J \mathrm{~B}_{p}^{\mathrm{T}} \mathrm{m}\right] \overline{\mathrm{S}}^{\mathrm{T}} \overline{\mathrm{B}}_{q}^{0} d V \\
& +\frac{2}{3} \int_{\Omega^{e}} \alpha\left(\overline{\mathrm{B}}_{p}^{0}\right)^{\mathrm{T}} \overline{\mathrm{S}} \theta\left[J \mathrm{~m}^{\mathrm{T}} \mathrm{B}_{q}\right] d V \\
& +\boldsymbol{I} \int_{\Omega^{e}} \alpha{ }^{2}\left(\boldsymbol{\nabla}_{0} \phi_{p}\right)^{\mathrm{T}} \overline{\boldsymbol{S}}\left(\boldsymbol{\nabla}_{0} \phi_{q}\right) d V
\end{aligned}
$$

\section{Numerical integration}

The cell-based integration of discrete quantities that depend on meshfree basis functions derivatives introduces integration errors when standard Gauss integration is used. To alleviate these integration errors in the VANP method, the second-order integration correction presented in Duan et al. [40, 41] is adopted. This approach satisfies the quadratic patch test and thus provides the regularity and resolution that is needed, and leads to a robust method in the large deformation regime. 
The numerical integration procedure needs an integration cell that is obtained from a simplicial tessellation. Fig. 3 depicts a typical tessellation and a representative integration cell in two dimensions for this scheme; the enhanced node set $\mathcal{N}^{+}$is also shown to remark that the nodal basis functions derivatives are to be computed using the enhanced node set since they stem from the displacement field.

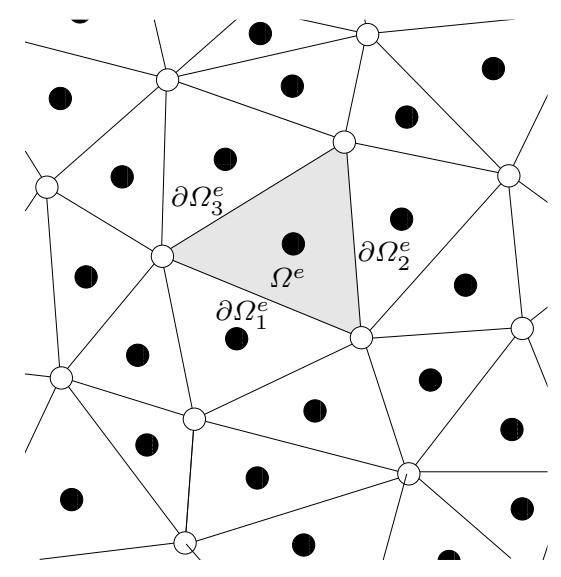

(a)

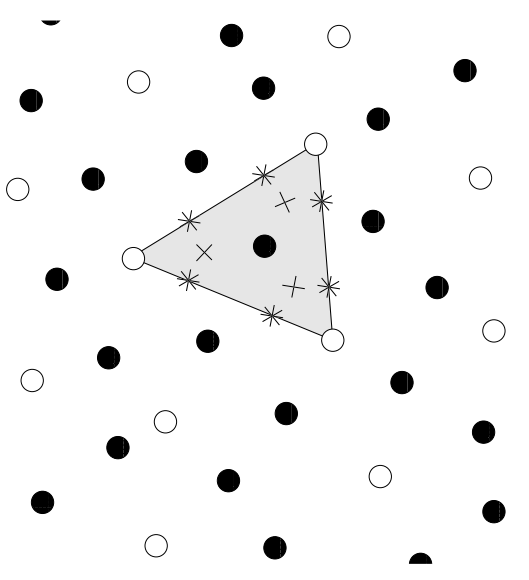

(b)

Fig. 3: Geometric entities for the second-order accurate integration scheme. (a) Simplicial tessellation, where the shaded region represents an integration cell whose domain is denoted by $\Omega^{e}$ and its boundary by $\partial \Omega^{e}=\partial \Omega_{1}^{e} \bigcup \partial \Omega_{2}^{e} \bigcup \partial \Omega_{3}^{e}$; and (b) the integration cell and nodes, where the interior Gauss points are depicted as + and the boundary Gauss points as $*$. Note that depending on the support size of the nodal basis functions, nodes that are beyond the cell can contribute at a Gauss point if their basis functions take a nonzero value at that point.

The standard second-order accurate Gauss integration scheme has three interior Gauss points on a triangular cell and four interior Gauss points on a tetrahedral cell. Essentially, the integration method to be used herein provides a correction to the values of the basis functions derivatives at these interior Gauss points. 
The Cartesian coordinate system is chosen, where for convenience $X \equiv X_{1}, Y \equiv$ $X_{2}$ and $Z \equiv X_{3}$. In addition, $n_{j}(j=1,2,3)$ is the $j$-th component of the unit outward normal to a cell edge in the Cartesian coordinate system. The second-order integration accuracy is obtained by requiring the basis functions derivatives to satisfy the divergence constraint

$$
\int_{\Omega^{e}} \phi_{a, j} f(\boldsymbol{X}) d V=\int_{\partial \Omega^{e}} \phi_{a} f(\boldsymbol{X}) n_{j} d S-\int_{\Omega^{e}} \phi_{a} f_{, j}(\boldsymbol{X}) d V,
$$

where $f(\boldsymbol{X})$ consists of zeroth- and first-order monomials in $\mathbb{R}^{d}$ :

$$
f(\boldsymbol{X})=\left[\begin{array}{llll}
1 & X_{1} & \ldots & X_{d}
\end{array}\right]^{\mathrm{T}} .
$$

The derivative $f_{, j}(\boldsymbol{X})$ ( $\delta_{i j}$ is the Kronecker delta symbol) is

$$
f_{, j}(\boldsymbol{X})=\left[\begin{array}{llll}
0 & \delta_{1 j} & \ldots & \delta_{d j}
\end{array}\right]^{\mathrm{T}} .
$$

On substituting (62) and (63) into (61) and using Gauss integration to numerically integrate the volume and surface integrals of the divergence constraint (see Fig. 3 for a two-dimensional representation of an integration cell) leads to the following system of linear equations:

$$
\mathbf{W d}_{j}=\mathbf{f}_{j}, \quad j=1,2,3
$$

where $\mathbf{W}$ and $\mathbf{f}_{j}$ are given in Ref. [38] (see (32) therein) for two-dimensions and in Ref. [41] (see (43) therein) for three-dimensions; the solution vector of the $j$-th basis function derivative evaluated at the interior Gauss points of the integration cell is:

$$
\mathrm{d}_{j}=\left[\begin{array}{lll}
\phi_{a, j}\left(\boldsymbol{X}_{1}\right) & \phi_{a, j}\left(\boldsymbol{X}_{2}\right) & \phi_{a, j}\left(\boldsymbol{X}_{3}\right)
\end{array}\right]^{\mathrm{T}}
$$

in two dimensions, and

$$
\mathrm{d}_{j}=\left[\begin{array}{llll}
\phi_{a, j}\left(\boldsymbol{X}_{1}\right) & \phi_{a, j}\left(\boldsymbol{X}_{2}\right) & \phi_{a, j}\left(\boldsymbol{X}_{3}\right) & \phi_{a, j}\left(\boldsymbol{X}_{4}\right)
\end{array}\right]^{\mathrm{T}}
$$


in three-dimensions, where $\boldsymbol{X}_{i}$ is the $i$-th interior Gauss point of the integration cell. In the preceding equations, the index a runs through the combined nodal contribution $^{2}$ that results from the union of nodal contributions corresponding to each of the interior and surface Gauss points in the cell.

The corrected derivatives given in (65) are used as the basis functions derivatives that appear in the stiffness matrices and residual nodal force vector of the VANP method.

\section{Numerical examples}

In this section, the performance of the two- and three-dimensional VANP formulation in the nearly-incompressible finite strain elastic regime is studied. To this end, the VANP method is compared to its closest finite element counterpart, the MINI element [1], which is inf-sup stable and is known to be the most stable finite element for the analysis of incompressible elastic solids at finite strains [5]. For some of the tests, the numerical solution of the standard displacement-based three-node triangular finite elements (FEM-T3) is also reported.

The base triangular or tetrahedral background mesh, which only contains the node set $\mathcal{N}^{s}$, is generated using GiD [52]. The enhanced node set $\mathcal{N}^{+}$is constructed when needed by adding the extra required nodes to the standard node set $\mathcal{N}^{s}$.

The numerical integration is performed using the approach presented in Section 5 that is based on the second-order accurate integration scheme of Duan et al. [40, 41]. Later in this section, a study is presented where the need to use this higher-order integration scheme is justified.

\footnotetext{
${ }^{2}$ The nodal contribution at a given Gauss point with coordinate $\boldsymbol{X}$ is defined as the indices of the nodes whose basis functions have a nonzero value at $\boldsymbol{X}$.
} 


\subsection{Nonlinear Cook's membrane}

This benchmark problem is used to study the behavior of nearly-incompressible formulations under combined bending and shear in distorted meshes [26, 27, 53]. The geometry and boundary conditions are shown in Figure 4(a). The left edge of the membrane is clamped and its right end is subjected to a deformation-independent shear load $P=6.25 \mathrm{~N} / \mathrm{mm}$ (total shear load of $100 \mathrm{~N}$ ). A regular background mesh of 3-node triangles with a mesh pattern of $n \times n$ subdivisions is chosen for the node set $\mathcal{N}^{s}$. A reference mesh for $n=20$ is shown in Fig. 4(b). The unstructured background mesh depicted in Fig. 4(c) is also considered for some tests. The strain energy function used for the hyperelastic material is:

$$
\Psi=\frac{1}{2} \mu\left(J^{-2 / 3} \operatorname{tr} C-3\right)+\frac{1}{2} \kappa\left(\frac{1}{2}\left(J^{2}-1\right)-\ln J\right)
$$

where the first term on the right hand side is the isochoric part and the second term the volumetric part. The material parameters are chosen as $\kappa=400942 \mathrm{MPa}$ and $\mu=80.1938 \mathrm{MPa}$, which represents a nearly-incompressible setting with Poisson's ratio of $\nu=0.4999$.

First, the convergence of the vertical tip displacement at point $A$ with mesh refinement is studied. The results are summarized in Fig. 5. The numerical results reveal that the VANP approach is in good agreement with the reference value given in Ref. [27] and delivers better convergence than the MINI element. The convergence plot also presents the standard FEM-T3 solution, where its expected locking behavior is evident.

Lastly, the smoothness of the nodal pressure obtained by the VANP formulation is assessed. Here, the unstructured mesh depicted in Fig. 4(c) is used. The result is provided in Fig. 6, where it is observed that the MINI element solution behaves somewhat oscillatory, whereas the VANP solution is smooth. 


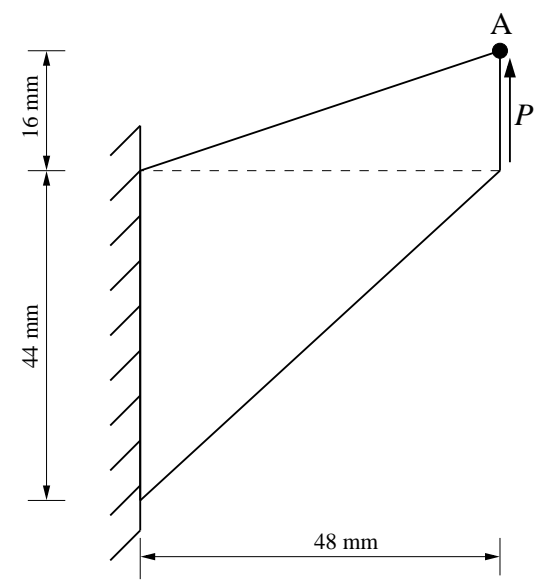

(a)

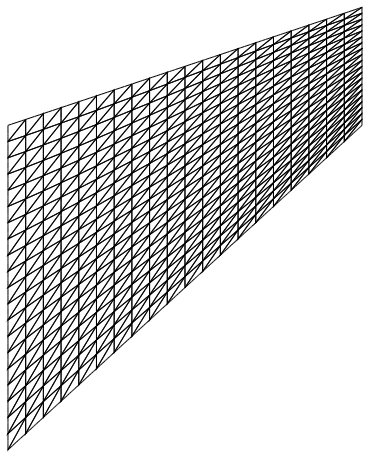

(b)

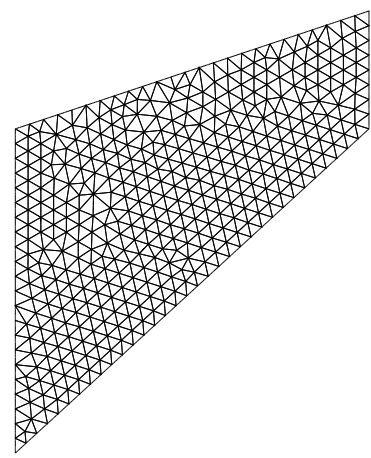

(c)

Fig. 4: Nonlinear Cook's membrane. (a) Geometry and boundary conditions; (b) sample regular background mesh; and (c) unstructured background mesh.

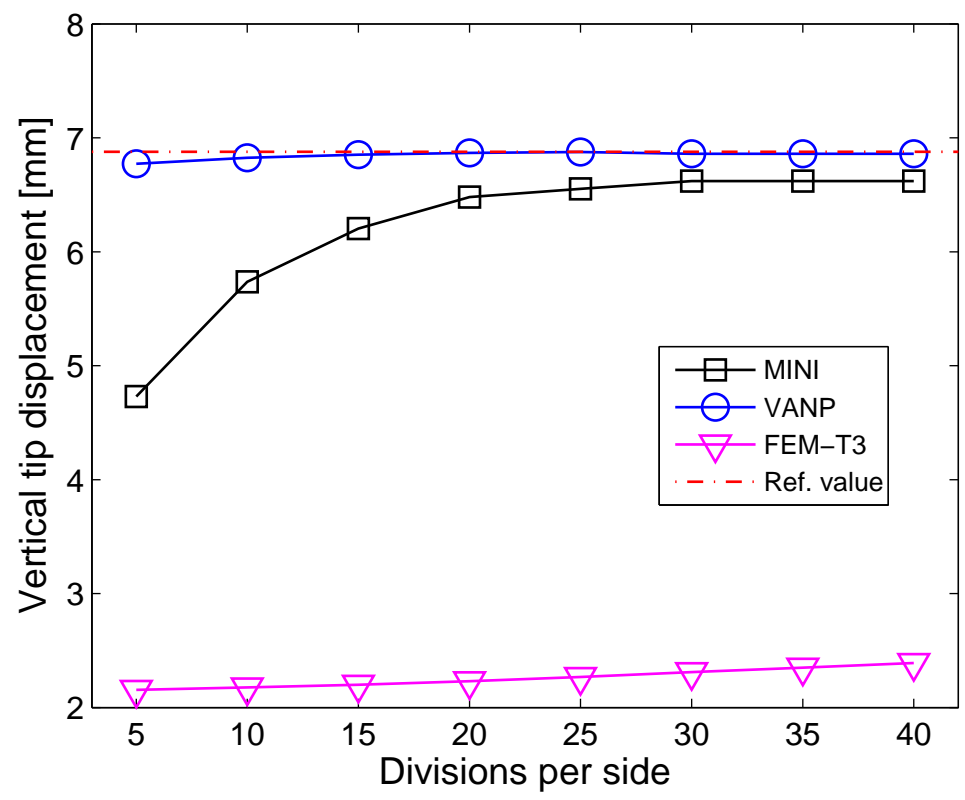

Fig. 5: Nonlinear Cook's membrane. Convergence of the vertical tip displacement at point A. 


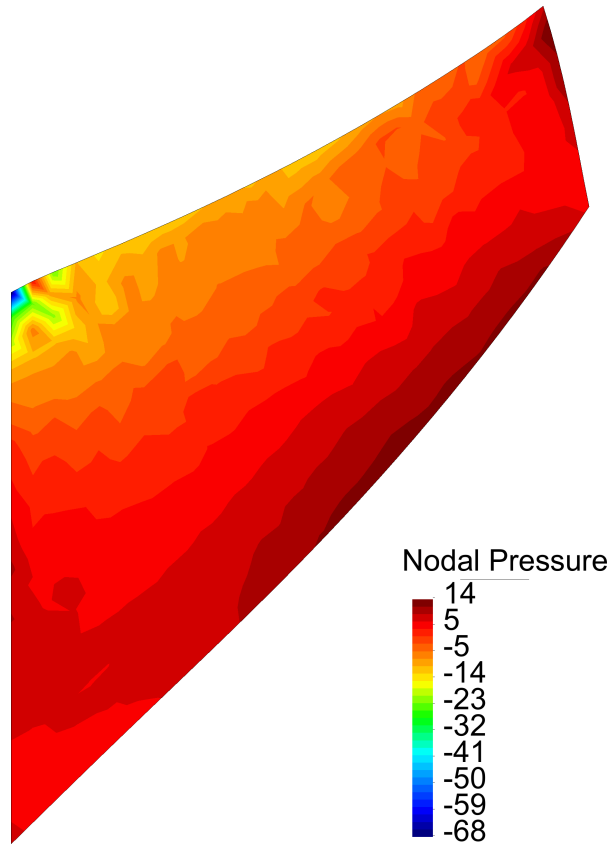

(a)

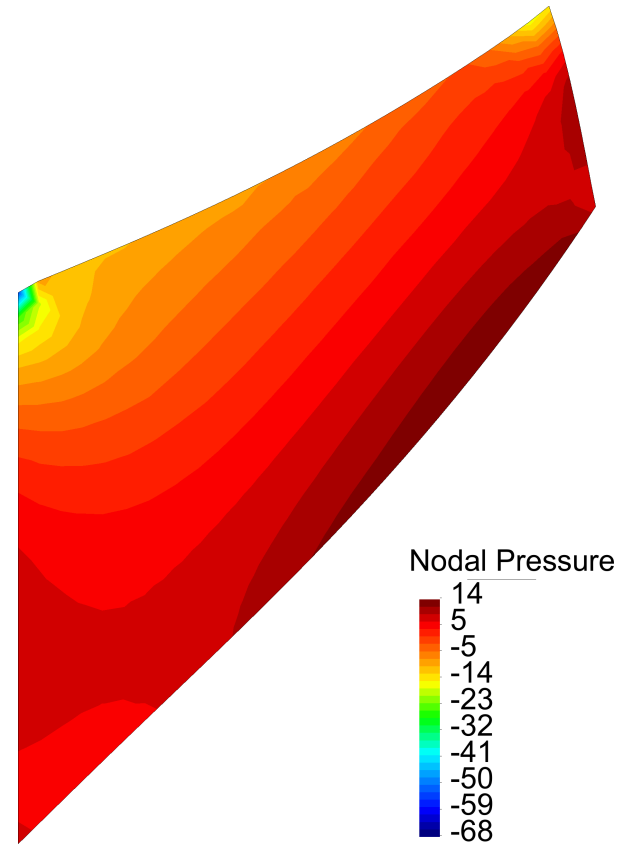

(b)

Fig. 6: Nonlinear Cook's membrane. Nodal pressure variable on the unstructured background mesh for (a) MINI element and (b) VANP formulation.

\subsection{Plane strain compression}

The following example is a standard test to demonstrate the ability of formulations for nearly-incompressible hyperelastic materials to withstand very large deformations in two dimensions [54] (plane strain condition is assumed) under the action of a deformation-independent compressive load $P$. The geometry and boundary conditions are shown in Fig. 7(a). The essential boundary conditions must be read as follows: the movement of the top surface is constrained along the horizontal direction and the movement of the bottom surface is constrained along the vertical direction. Due to the symmetry of the problem, only one-half of the model is discretized with a background mesh like the one depicted in Fig. 7(b). The hyperelastic material is 
defined using the following strain energy function:

$$
\Psi=\frac{1}{2} \mu\left(J^{-2 / 3} \operatorname{tr} C-3\right)-\mu \ln J+\frac{1}{4} \kappa\left(J^{2}-1-2 \ln J\right) .
$$

The material parameters are set as $\kappa=400889.806 \mathrm{MPa}$ and $\mu=80.194 \mathrm{MPa}$, which represents a nearly-incompressible setting with Poisson's ratio of $\nu=0.4999$.

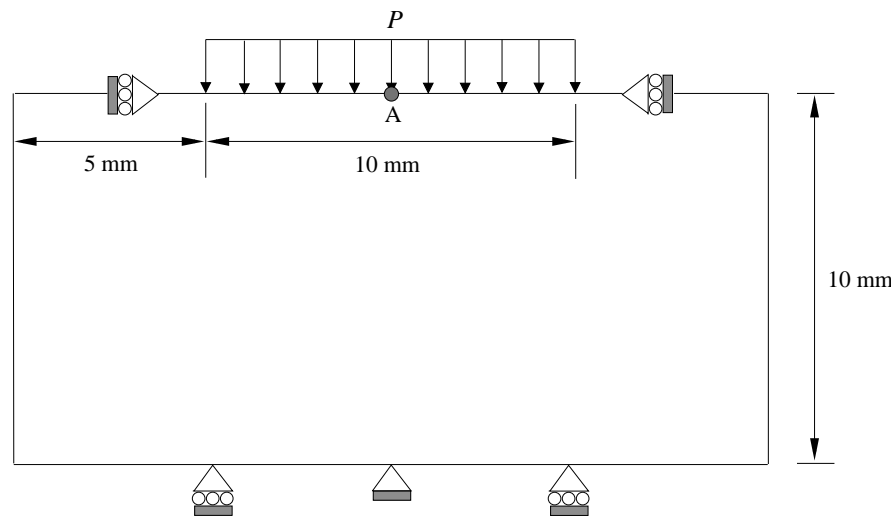

(a)

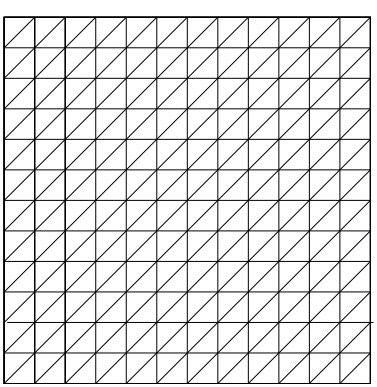

(b)

Fig. 7: Plane strain compression. (a) Model geometry and boundary conditions; and (b) reference background mesh for one half of the model.

First, a convergence study is conducted. For this, the compression level is defined as $\left|u_{A}\right| / h \times 100$ with $u_{A}$ being the vertical displacement at point $A$ and $h$ the height of the block (see Fig. 7(a)). Fig. 8 presents the convergence of the compression level upon mesh refinement for different values of $P$ in $\mathrm{MPa}$. The results are available for the MINI element and VANP formulation. It is observed that the converged values of the compression level delivered by the VANP formulation are in good agreement with those provided in Ref. [54], whereas those for the MINI element are higher than the reference value in all the cases.

Finally, the smoothness of the VANP method is once again evident in Fig. 9, where 


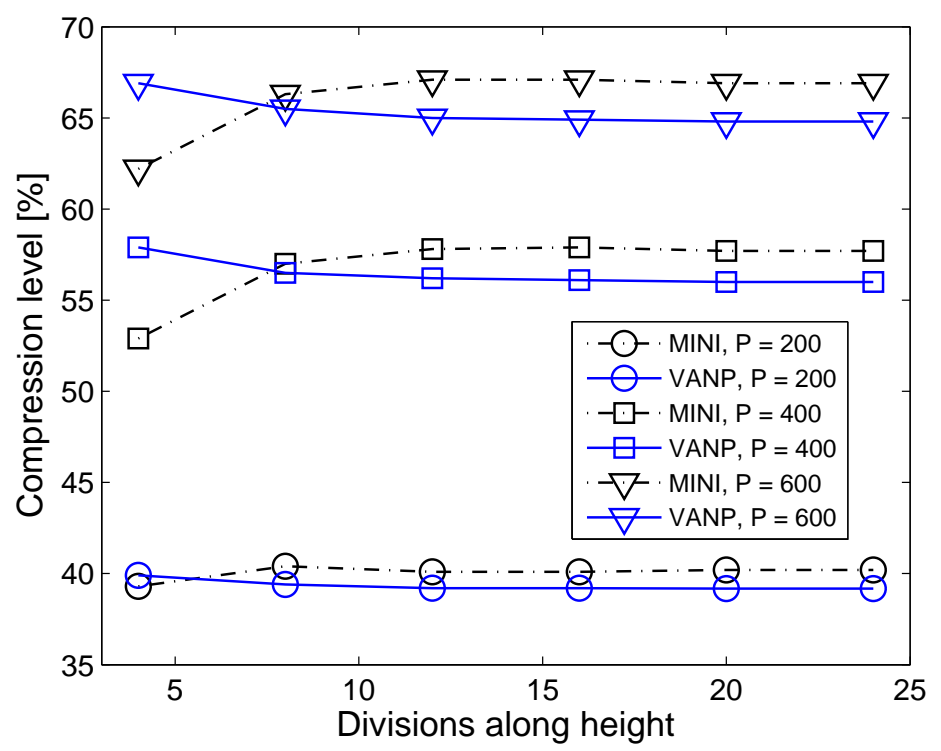

Fig. 8: Plane strain compression. Compression level for different values of the pressure on the top surface.

it is observed that the distortion of the elements does not affect the smoothness of the nodal pressure field. The same is not true for the MINI element.

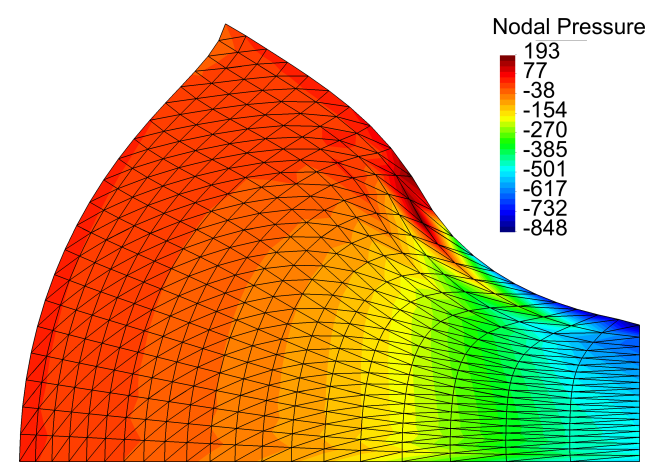

(a)

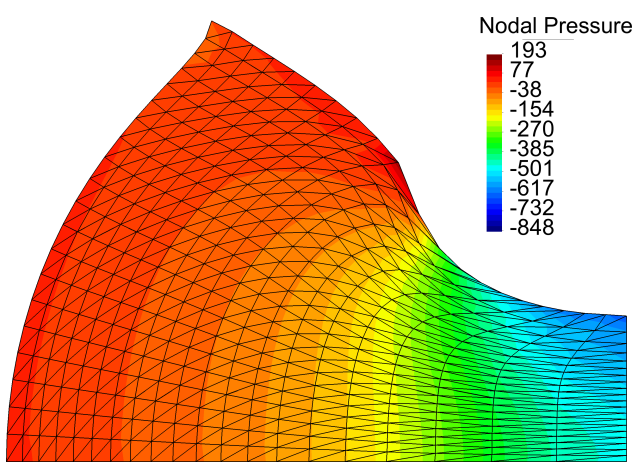

(b)

Fig. 9: Plane strain compression. Nodal pressure variable for (a) MINI element and (b) VANP formulation. 


\subsection{Three-dimensional cantilever beam}

This example considers a three-dimensional bending-dominated problem, where a beam is clamped at one end and subjected to a deformation-independent transverse uniformly distributed load on the other end. Fig. 10 illustrates the problem setup. The node set $\mathcal{N}^{s}$ is obtained from a mesh of pattern $n \times n / 2 \times(n / 2-1)$ divisions, where $n$ is the number of divisions along the length of the beam and $n / 2$ the number of divisions along its height. The following strain energy function is used to represent the nearly-incompressible hyperelastic material with bulk modulus $\kappa=166666666.67 \mathrm{~Pa}$ and shear modulus $\mu=333555.704 \mathrm{~Pa}(\nu=0.499)$ :

$$
\Psi=\frac{1}{2} \mu\left(J^{-2 / 3} \operatorname{tr} C-3\right)+\frac{1}{2} \kappa(J-1)^{2},
$$

where the first term on the right hand side is the isochoric part and the second term is the volumetric part.

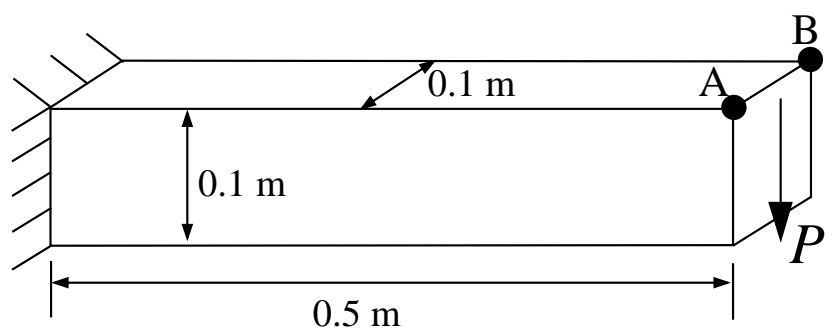

Fig. 10: Three-dimensional cantilever beam. Model geometry and boundary conditions.

We start by studying the convergence of the downward tip displacement on edge $\overline{\mathrm{AB}}$ upon mesh refinement. In this study, three values for the transverse load are considered: $P=5000 \mathrm{~N} / \mathrm{m}, P=10000 \mathrm{~N} / \mathrm{m}$ and $P=15000 \mathrm{~N} / \mathrm{m}$. The convergence study is presented in Fig. 11 for both the MINI element and the VANP methods. Reference solutions are obtained from a converged mesh of 27-node $u$ - $p$ brick finite elements. It is observed that the VANP solutions converge to the reference values, 
whereas the MINI element behaves quite 'stiff' in this bending-dominated problem and convergence is difficult to attain to reach the total number of Newton load steps as the mesh is refined - the missing data for the MINI element in the convergence plot represent this issue.

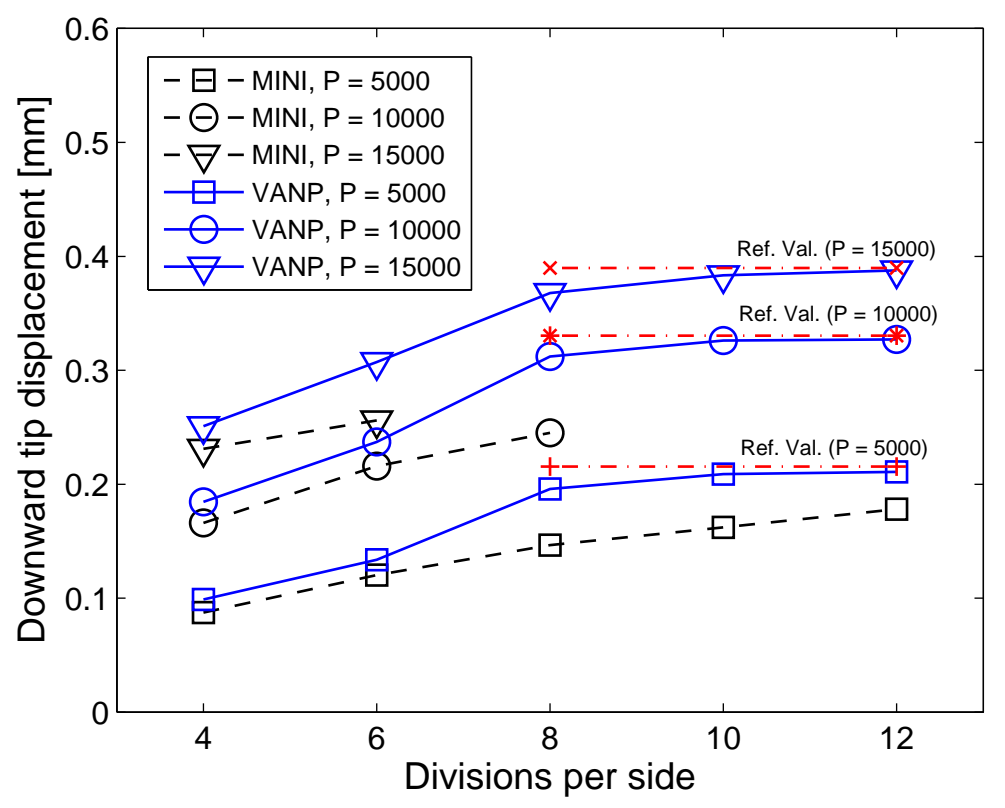

Fig. 11: Three-dimensional cantilever beam. Convergence of the downward tip displacement on edge $\overline{\mathrm{AB}}$.

Lastly, the vertical displacement and nodal pressure fields that are delivered by the MINI element and the VANP methods are presented in the pictorial shown in Fig. 12. The plots are obtained for $n=8$ and $P=10000 \mathrm{~N} / \mathrm{m}$. In comparing the nodal pressure fields, these plots reveal that the VANP method delivers smoother pressure fields than the MINI element method. The 'stiff' behavior of the MINI element is also evident in these plots. 


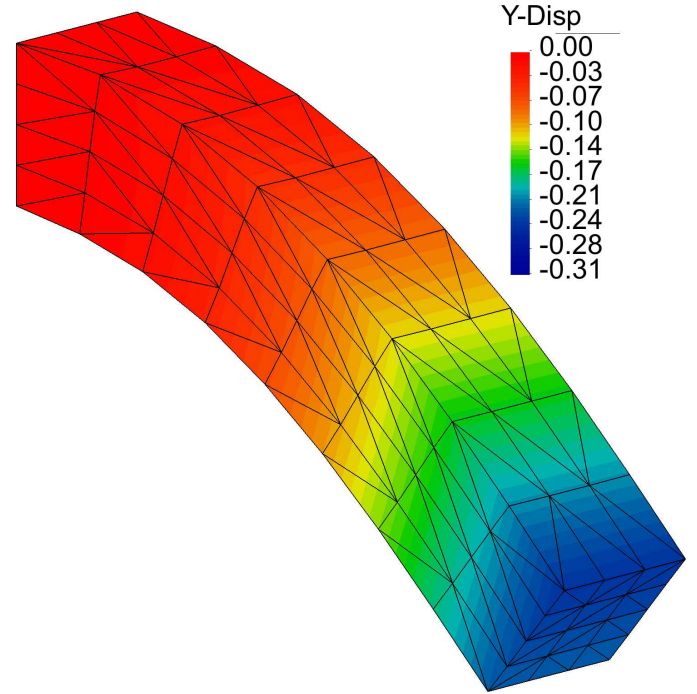

(a)

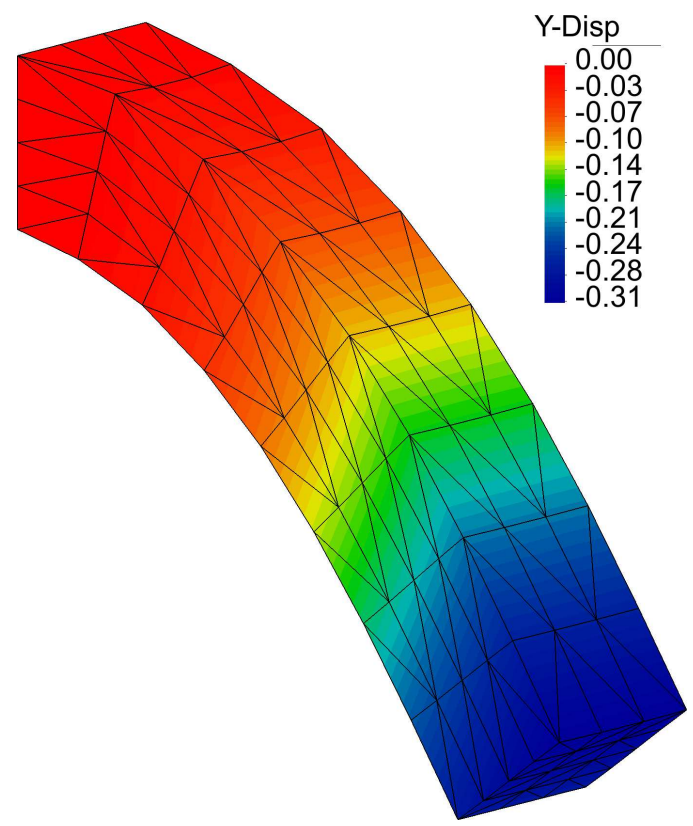

(c)

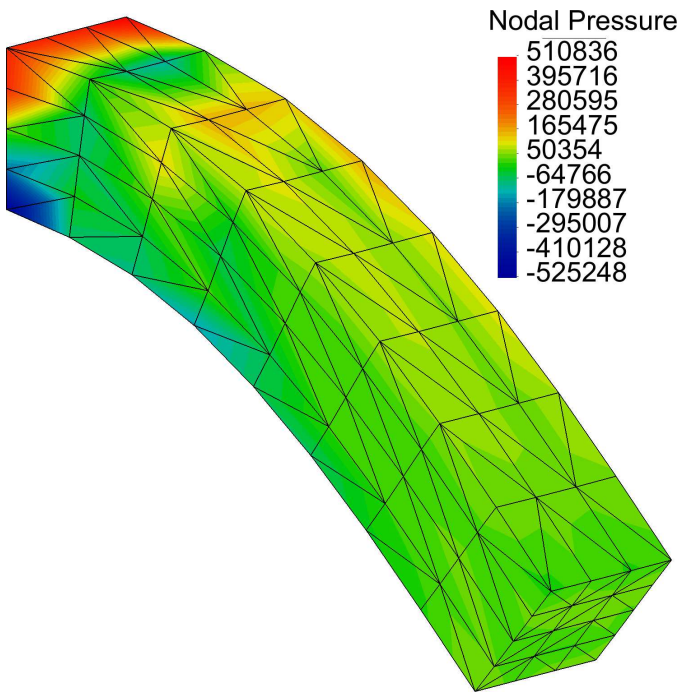

(b)

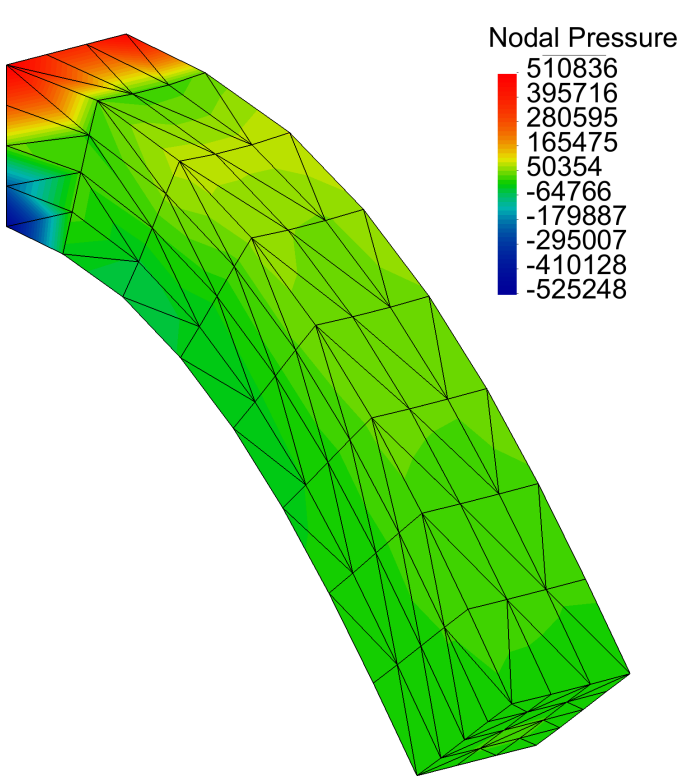

(d)

Fig. 12: Nodal field variables for the three-dimensional cantilever beam problem. (a) Vertical displacement delivered by the MINI element method, (b) nodal pressure delivered by the MINI element method, (c) vertical displacement delivered by the VANP method, and (d) nodal pressure delivered by the VANP method. 


\subsection{Three-dimensional compression}

A three-dimensional analysis of a hyperelastic rubber block under the action of a deformation-independent compressive load $P$ is considered. The geometry, boundary conditions and a reference regular background mesh are shown in Fig. 13. The strain energy function of the hyperelastic material used is:

$$
\Psi=\frac{1}{2} \mu\left(J^{-2 / 3} \operatorname{tr} C-3\right)-\mu \ln J+\frac{1}{2} \kappa(\ln J)^{2} .
$$

The material parameters are chosen as $\kappa=400889.806 \mathrm{MPa}$ and $\mu=80.194 \mathrm{MPa}$, which represents a nearly-incompressible setting with Poisson's ratio of $\nu=0.4999$.

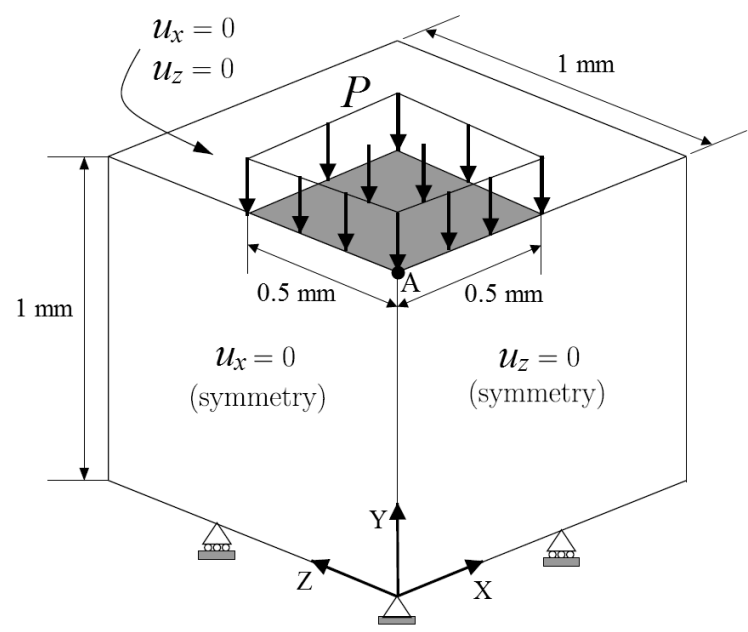

(a)

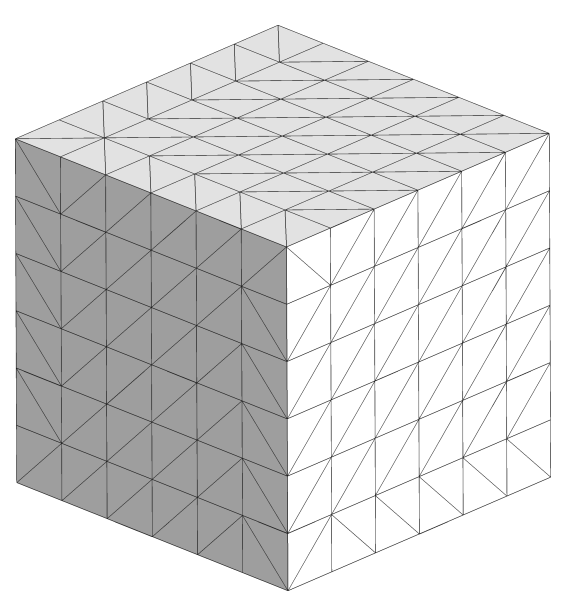

(b)

Fig. 13: Three-dimensional compression. (a) Model geometry and boundary conditions and (b) reference regular background mesh.

The starting study consists in a convergence test. To this end, we define the compression level as $\left|u_{A}\right| / h \times 100$, where $u_{A}$ is the vertical displacement at point $A$ and $h$ the height of the block (see Fig. 13(a)). The convergence of the compression level upon mesh refinement for different values of $P$ in $\mathrm{MPa}$ is presented in Fig. 14 for the 
MINI element and VANP formulation. It is observed that the converged values of the compression level that are delivered by the VANP formulation are in good agreement with those provided in Refs. $[27,55]$. On the other hand, the MINI element only performs well for the lowest compressive load, whereas for the higher compressive loads convergence is difficult to reach the total number of Newton load steps as the mesh is refined.

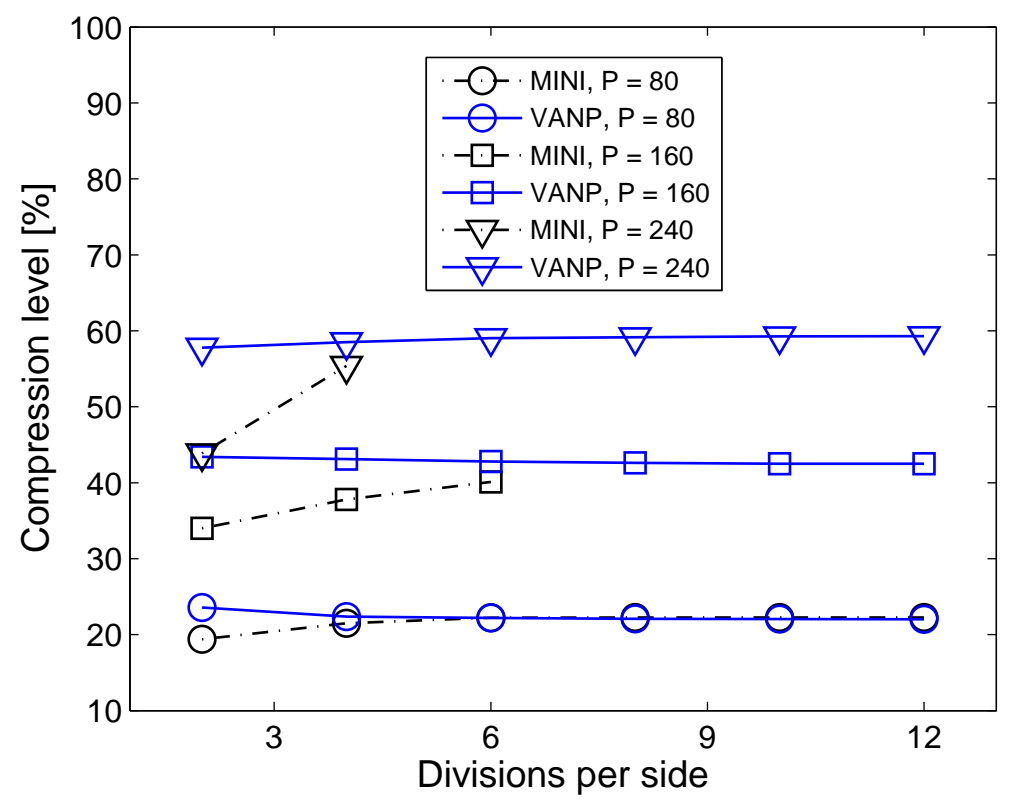

Fig. 14: Three-dimensional compression. Compression level for different values of the pressure on the top surface.

To demonstrate the need for a modified higher-order integration scheme, we solve the three-dimensional compression problem using the following integration schemes: first-order standard Gauss integration, second-order standard Gauss integration, fifth-order standard Gauss integration, integration scheme of Duan et al. [40] that is based on Ref. [31], second-order modified integration of Ref. [25], and 
finally the second-order modified integration of Duan et al. [41]. For each scheme, the effect of the numerical integration on the nodal pressure field is presented in Fig. 15. Figs. 15(a)-(e) reveal severe pressure oscillations, and Figs. 15(a)-(b) and 15(d) also depict nonsmooth deformations on the top surface. Fig. 15(f) reveals that the second-order accurate modified integration scheme of Duan et al. [41] provides a robust solution, with smooth deformation and pressure fields.

In closing this section, the performance of the VANP method using an unstructured background mesh is demonstrated. Fig. 16 shows the mesh used in the test. The VANP solution is compared to the solution delivered by the MINI element for $P=240 \mathrm{MPa}$. The solution of the vertical displacement is depicted in Fig. 17 for both methods. The MINI element cannot undergo the total number of Newton load steps, thus only its last converged solution is presented in Fig. 17(a). On the contrary, the VANP formulation exhibits no difficulty in running through the total number of Newton load steps as inferred from Fig. 17(b), where a compression level of around $59 \%$ is achieved. This value is in complete agreement with the one expected from Fig. 14 . The nodal pressure field is shown in Fig. 18, where pressure oscillations are observed for the MINI element. On the contrary, the nodal pressure solution delivered by the VANP formulation is smooth.

\section{Concluding Remarks}

A projection scheme for meshfree methods, which we refer to as the volumeaveraged nodal projection (VANP) method, has been proposed for the analysis of nearly-incompressible elastic solids at finite strains. In this approach, a volumeaveraged nodal projection operator is constructed to average the dilatational constraint at a node from the displacement field of surrounding nodes. The nodal dilatational constraint is then projected onto the linear approximation space. The 


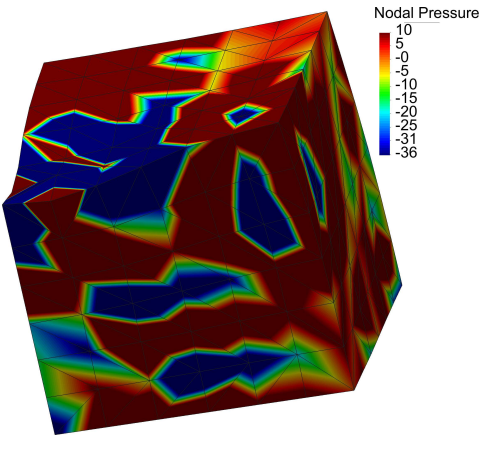

(a)

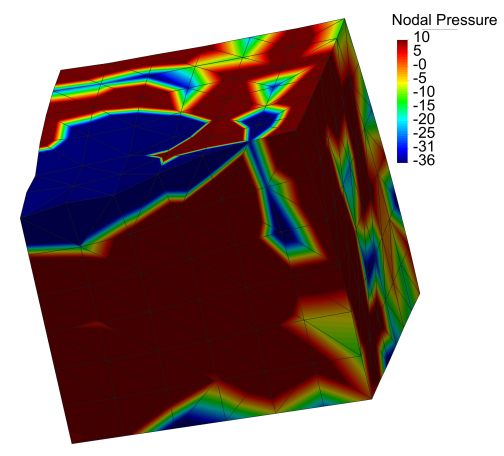

(d)

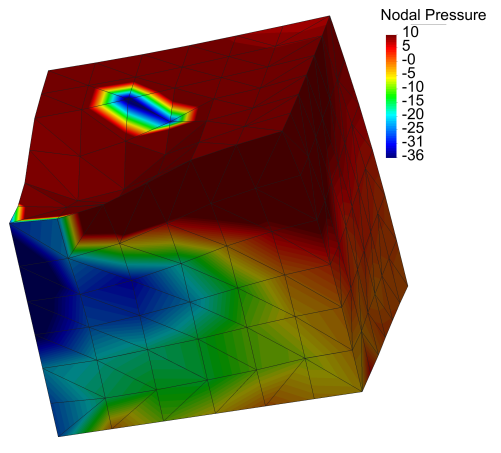

(b)

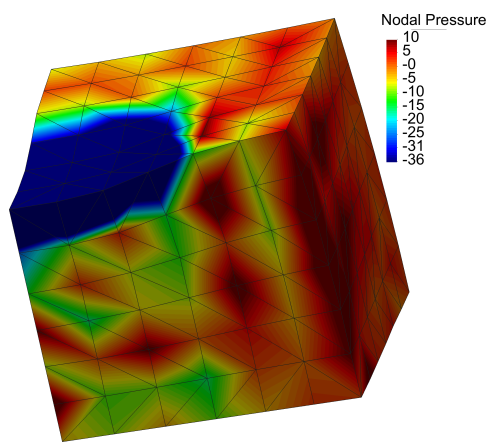

(e)

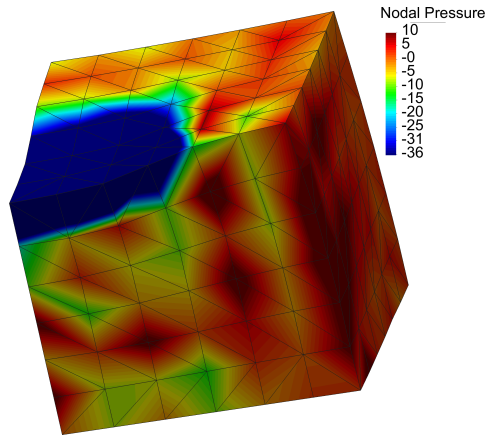

(c)

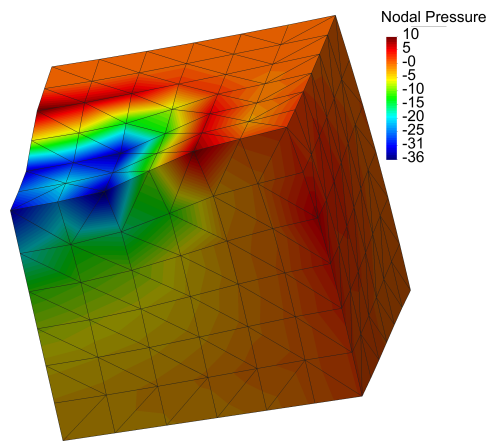

(f)

Fig. 15: Effect of numerical integration on the nodal pressure field for the three-dimensional compression test problem. (a) first-order standard Gauss integration, (b) second-order standard Gauss integration, (c) fifth-order standard Gauss integration, (d) integration scheme of Duan et al. [40] that is based on Ref. [31], (e) second-order modified integration of Ref. [25] and (f) second-order modified integration of Duan et al. [41]. The plots show that the inaccuracies are alleviated by the integration scheme of Duan et al. [41].

displacement field is constructed on the linear space and enriched with bubble-like meshfree basis functions for stability, which mimics the inf-sup stable MINI [1] finite element. The projection operator permits to formulate the problem as a function of only the displacement field, which makes the VANP formulation a displacement-based approach. The nodal information for the computation of the meshfree basis functions 


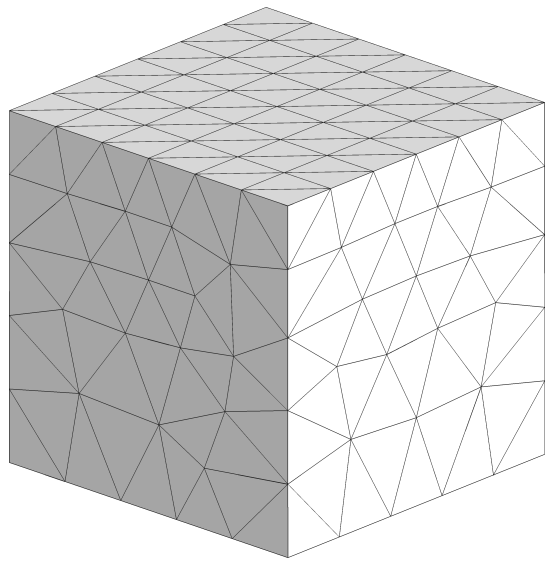

(a)

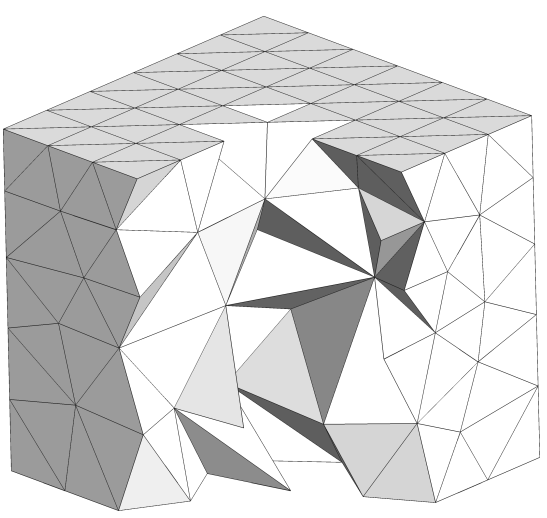

(b)

Fig. 16: Three-dimensional compression. Unstructured background mesh: (a) exterior view and (b) internal view.

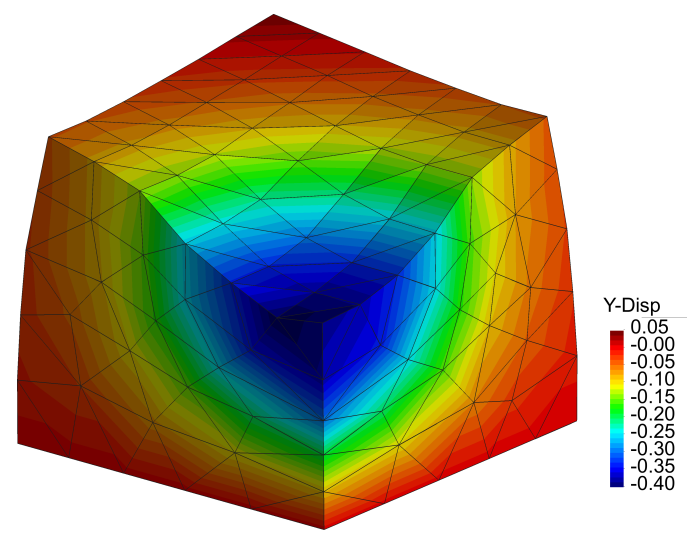

(a)

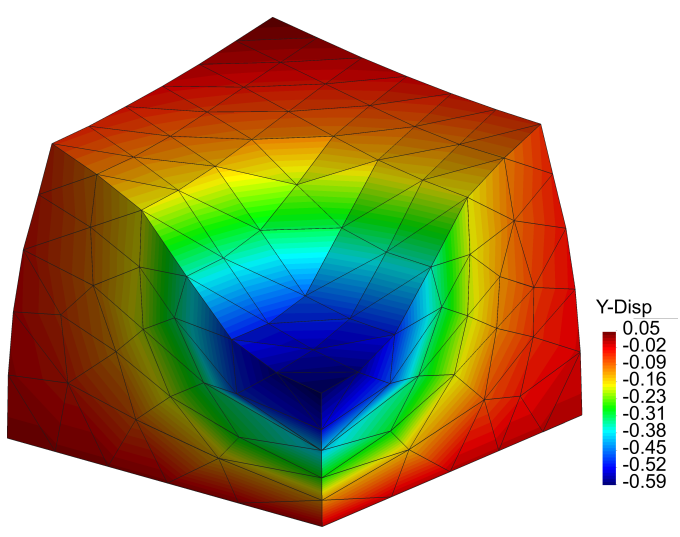

(b)

Fig. 17: Three-dimensional compression. Vertical displacement solution for (a) MINI element and (b) VANP formulation. In contrast to the VANP method, the MINI element cannot go through the total number of Newton's load steps.

is obtained from a background mesh of three-node triangles or four-node tetrahedra, which also serves for the numerical integration of the weak form integrals. Numeri- 


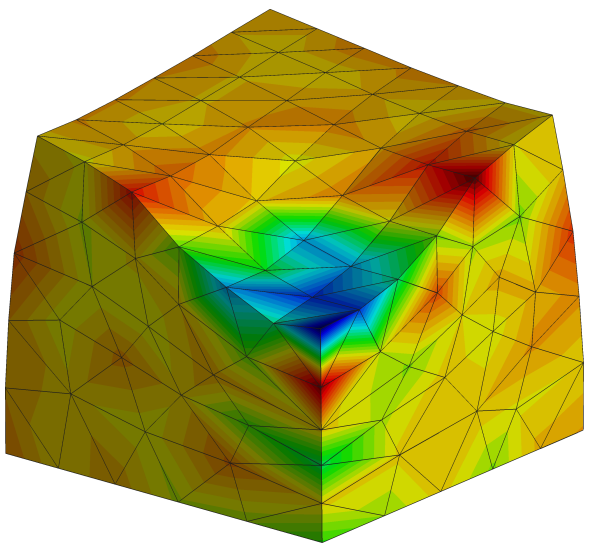

(a)

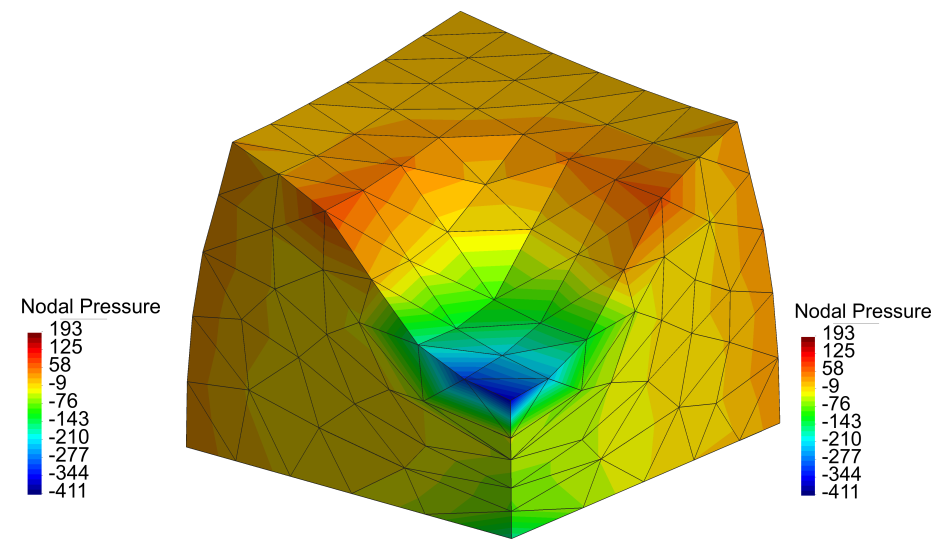

(b)

Fig. 18: Three-dimensional compression. Nodal pressure solution for (a) MINI element and (b) VANP formulation. The MINI element exhibits pressure oscillations, whereas the VANP solution is smooth.

cal integration inaccuracies to which meshfree methods are prone are tackled using a modified Gauss integration scheme based on the second-order accurate integration rule of Duan et al. [40, 41].

Four benchmark problems which included the nonlinear Cook's membrane, a plane strain and a three-dimensional compression of a rubber block, and a threedimensional cantilever beam were studied to demonstrate the performance of the VANP method in nearly-incompressible analysis of nonlinear elastic solids using loworder triangular and tetrahedral background meshes. The numerical solutions delivered by the VANP method were compared to those of the MINI element, which is its closest finite element counterpart for low-order tessellations. The numerical examples in two-dimensions showed that the VANP formulation provides faster convergence and smoother pressure fields than the MINI element formulation. Furthermore, the superiority of the VANP formulation over the MINI element formulation is also established in three-dimensions, where the former allows tetrahedral background meshes to 
achieve larger deformations with smooth pressure fields. In conclusion, the proposed methodology provides improved robustness for nearly-incompressible nonlinear large deformation analysis on simplicial (Delaunay) tessellations.

\section{Appendix A. Directional derivative of the modified Green-Lagrange strain tensor}

The detailed derivation of (40) is developed. The following equations are obtained from the standard literature (for instance, see Ref. [47]):

$$
\begin{gathered}
\mathrm{D} \boldsymbol{F}(\chi)[\boldsymbol{v}]=\nabla_{0} \boldsymbol{v}, \\
\mathrm{D} J(\chi)[\boldsymbol{v}]=J \operatorname{tr}\left(\boldsymbol{\nabla}_{0} \boldsymbol{v} \boldsymbol{F}^{-1}\right) .
\end{gathered}
$$

We now consider (A.2) and proceed to take the directional derivative of (32), which yields

$$
\mathrm{D} J_{c}(\chi)[\boldsymbol{v}]=\frac{\int_{\Omega_{c}} \phi_{c} J \operatorname{tr}\left(\boldsymbol{\nabla}_{0} \boldsymbol{v} \boldsymbol{F}^{-1}\right) d V}{\int_{\Omega_{c}} \phi_{c} d V} .
$$

On taking the directional derivative of (34) and using (A.3) leads to

$$
\mathrm{D} \bar{J}(\chi)[\boldsymbol{v}]=\pi\left[J \operatorname{tr}\left(\boldsymbol{\nabla}_{0} \boldsymbol{v} \boldsymbol{F}^{-1}\right)\right] .
$$

The directional derivative of $\alpha=(\bar{J} / J)^{1 / 3}$ is developed with the aid of (A.4) and the operator defined in (39) as follows:

$$
\begin{aligned}
\mathrm{D} \alpha(\chi)[\boldsymbol{v}] & =\frac{1}{3 \alpha^{2}}\left[\frac{1}{J} \mathrm{D} \bar{J}(\chi)[\boldsymbol{v}]-\frac{\bar{J}}{J^{2}} \mathrm{D} J(\chi)[\boldsymbol{v}]\right] \\
& =\frac{1}{3 \alpha^{2} J}\left[\pi\left[J \operatorname{tr}\left(\boldsymbol{\nabla}_{0} \boldsymbol{v} \boldsymbol{F}^{-1}\right)\right]-\bar{J} \operatorname{tr}\left(\boldsymbol{\nabla}_{0} \boldsymbol{v} \boldsymbol{F}^{-1}\right)\right] \\
& =\frac{1}{3} \alpha \theta\left[J \operatorname{tr}\left(\boldsymbol{\nabla}_{0} \boldsymbol{v} \boldsymbol{F}^{-1}\right)\right] .
\end{aligned}
$$

In addition, the directional derivative of (36) is:

$$
\mathrm{D} \overline{\boldsymbol{E}}(\chi)[\boldsymbol{v}]=\frac{1}{2}\left[(\mathrm{D} \overline{\boldsymbol{F}}(\chi)[\boldsymbol{v}])^{\mathrm{T}} \overline{\boldsymbol{F}}+\overline{\boldsymbol{F}}^{\mathrm{T}} \mathrm{D} \overline{\boldsymbol{F}}(\chi)[\boldsymbol{v}]\right] .
$$


We now make use of (24) in conjunction with (A.1) and (A.5) to write

$$
\begin{aligned}
\mathrm{D} \overline{\boldsymbol{F}}(\chi)[\boldsymbol{v}] & =\mathrm{D} \alpha(\chi)[\boldsymbol{v}] \boldsymbol{F}+\alpha \mathrm{D} \boldsymbol{F}(\chi)[\boldsymbol{v}] \\
& =\frac{1}{3} \alpha \theta\left[J \operatorname{tr}\left(\boldsymbol{\nabla}_{0} \boldsymbol{v} \boldsymbol{F}^{-1}\right)\right] \boldsymbol{F}+\alpha \boldsymbol{\nabla}_{0} \boldsymbol{v}
\end{aligned}
$$

whose substitution, together with (24), into (A.6) leads to the final expression for the modified Green-Lagrange strain tensor:

$$
\mathrm{D} \overline{\boldsymbol{E}}(\chi)[\boldsymbol{v}]=\frac{1}{3} \theta\left[J \operatorname{tr}\left(\boldsymbol{\nabla}_{0} \boldsymbol{v} \boldsymbol{F}^{-1}\right)\right] \overline{\boldsymbol{F}}^{\mathrm{T}} \overline{\boldsymbol{F}}+\alpha\left(\overline{\boldsymbol{F}}^{\mathrm{T}} \boldsymbol{\nabla}_{0} \boldsymbol{v}\right)_{\mathrm{sym}}
$$

\section{Appendix B. Second variation of the modified energy functional}

The detailed derivation of (43) is developed. The directional derivative of the modified second Piola-Kirchhoff stress tensor is first derived. In this process, we use (44) in conjunction with (A.8) and proceed as follows:

$$
\begin{aligned}
\mathrm{D} \overline{\boldsymbol{S}}(\chi)[\Delta \boldsymbol{u}] & =\frac{\partial \boldsymbol{S}(\boldsymbol{E})}{\partial \boldsymbol{E}}(\overline{\boldsymbol{E}}(\chi)): \mathrm{D} \overline{\boldsymbol{E}}(\chi)[\Delta \boldsymbol{u}] \\
& =\overline{\mathcal{D}}: \mathrm{D} \overline{\boldsymbol{E}}(\chi)[\Delta \boldsymbol{u}] \\
& =\frac{1}{3} \theta\left[J \operatorname{tr}\left(\boldsymbol{\nabla}_{0} \Delta \boldsymbol{u} \boldsymbol{F}^{-1}\right)\right] \overline{\mathcal{D}}:\left(\overline{\boldsymbol{F}}^{\mathrm{T}} \overline{\boldsymbol{F}}\right)+\alpha \overline{\mathcal{D}}:\left(\overline{\boldsymbol{F}}^{\mathrm{T}} \boldsymbol{\nabla}_{0} \Delta \boldsymbol{u}\right)_{\mathrm{sym}} \cdot(
\end{aligned}
$$

Next, the definition of the directional derivative of the inverse of a tensor [47] is used to write

$$
\mathrm{D} \boldsymbol{F}^{-1}(\chi)[\Delta \boldsymbol{u}]=-\boldsymbol{F}^{-1} \nabla_{0} \Delta \boldsymbol{u} \boldsymbol{F}^{-1}
$$

which is employed along with (A.2) in the derivation of the following directional derivative:

$$
\begin{aligned}
\mathrm{D}\left(J \operatorname{tr}\left(\boldsymbol{\nabla}_{0} \boldsymbol{v} \boldsymbol{F}^{-1}\right)\right)[\Delta \boldsymbol{u}] & =\mathrm{D} J(\chi)[\Delta \boldsymbol{u}] \operatorname{tr}\left(\boldsymbol{\nabla}_{0} \boldsymbol{v} \boldsymbol{F}^{-1}\right)+J \operatorname{tr}\left(\boldsymbol{\nabla}_{0} \boldsymbol{v} \mathrm{D} \boldsymbol{F}^{-1}(\chi)[\Delta \boldsymbol{u}]\right) \\
& =J\left[\operatorname{tr}\left(\boldsymbol{\nabla}_{0} \boldsymbol{v} \boldsymbol{F}^{-1}\right) \operatorname{tr}\left(\boldsymbol{\nabla}_{0} \Delta \boldsymbol{u} \boldsymbol{F}^{-1}\right)-\operatorname{tr}\left(\boldsymbol{\nabla}_{0} \boldsymbol{v} \boldsymbol{F}^{-1} \nabla_{0} \Delta \boldsymbol{u} \boldsymbol{F}^{-1}\right)\right]
\end{aligned}
$$


The directional derivatives of the operators that were defined in (31) and (39) follows. With the aid of (B.3) the following expression is obtained for the operator (31):

$$
\begin{aligned}
\mathrm{D} \pi\left(J \operatorname{tr}\left(\boldsymbol{\nabla}_{0} \boldsymbol{v} \boldsymbol{F}^{-1}\right)\right)[\Delta \boldsymbol{u}]= & \pi\left[\operatorname{Jtr}\left(\boldsymbol{\nabla}_{0} \boldsymbol{v} \boldsymbol{F}^{-1}\right) \operatorname{tr}\left(\boldsymbol{\nabla}_{0} \Delta \boldsymbol{u} \boldsymbol{F}^{-1}\right)\right] \\
& -\pi\left[J \operatorname{tr}\left(\boldsymbol{\nabla}_{0} \boldsymbol{v} \boldsymbol{F}^{-1} \boldsymbol{\nabla}_{0} \Delta \boldsymbol{u} \boldsymbol{F}^{-1}\right)\right],
\end{aligned}
$$

whereas (B.4) along with (A.2), (A.4) and (B.3) are used for the operator (39) to arrive at the following expression:

$$
\begin{aligned}
\mathrm{D} \theta\left[J \operatorname{tr}\left(\boldsymbol{\nabla}_{0} \boldsymbol{v} \boldsymbol{F}^{-1}\right)\right][\Delta \boldsymbol{u}]= & -\frac{1}{\bar{J}^{2}} \pi\left[J \operatorname{tr}\left(\boldsymbol{\nabla}_{0} \boldsymbol{v} \boldsymbol{F}^{-1}\right)\right] \pi\left[J \operatorname{tr}\left(\boldsymbol{\nabla}_{0} \Delta \boldsymbol{u} \boldsymbol{F}^{-1}\right)\right] \\
& +\frac{1}{\bar{J}} \pi\left[J \operatorname{tr}\left(\boldsymbol{\nabla}_{0} \boldsymbol{v} \boldsymbol{F}^{-1}\right) \operatorname{tr}\left(\boldsymbol{\nabla}_{0} \Delta \boldsymbol{u} \boldsymbol{F}^{-1}\right)\right] \\
& -\frac{1}{\bar{J}} \pi\left[J \operatorname{tr}\left(\boldsymbol{\nabla}_{0} \boldsymbol{v} \boldsymbol{F}^{-1} \boldsymbol{\nabla}_{0} \Delta \boldsymbol{u} \boldsymbol{F}^{-1}\right)\right] \\
& +\operatorname{tr}\left(\boldsymbol{\nabla}_{0} \boldsymbol{v} \boldsymbol{F}^{-1} \boldsymbol{\nabla}_{0} \Delta \boldsymbol{u} \boldsymbol{F}^{-1}\right) .
\end{aligned}
$$

Noting that $\mathrm{D}\left(\overline{\boldsymbol{F}}^{\mathrm{T}} \overline{\boldsymbol{F}}\right)[\boldsymbol{v}]=2 \mathrm{D} \overline{\boldsymbol{E}}[\boldsymbol{v}]$ and using (B.5), yields the following directional derivative:

$\mathrm{D}\left(\theta\left[J \operatorname{tr}\left(\boldsymbol{\nabla}_{0} \boldsymbol{v} \boldsymbol{F}^{-1}\right)\right] \overline{\boldsymbol{F}}^{\mathrm{T}} \overline{\boldsymbol{F}}\right)[\Delta \boldsymbol{u}]=-\frac{1}{\bar{J}^{2}} \pi\left[J \operatorname{tr}\left(\boldsymbol{\nabla}_{0} \boldsymbol{v} \boldsymbol{F}^{-1}\right)\right] \pi\left[J \operatorname{tr}\left(\boldsymbol{\nabla}_{0} \Delta \boldsymbol{u} \boldsymbol{F}^{-1}\right)\right] \overline{\boldsymbol{F}}^{\mathrm{T}} \overline{\boldsymbol{F}}$

$$
\begin{aligned}
& +\frac{1}{\bar{J}} \pi\left[J \operatorname{tr}\left(\nabla_{0} \boldsymbol{v} \boldsymbol{F}^{-1}\right) \operatorname{tr}\left(\nabla_{0} \Delta \boldsymbol{u} \boldsymbol{F}^{-1}\right)\right] \overline{\boldsymbol{F}}^{\mathrm{T}} \overline{\boldsymbol{F}} \\
& -\frac{1}{\bar{J}} \pi\left[J \operatorname{tr}\left(\boldsymbol{\nabla}_{0} \boldsymbol{v} \boldsymbol{F}^{-1} \boldsymbol{\nabla}_{0} \Delta \boldsymbol{u} \boldsymbol{F}^{-1}\right)\right] \overline{\boldsymbol{F}}^{\mathrm{T}} \overline{\boldsymbol{F}}
\end{aligned}
$$$$
+\operatorname{tr}\left(\boldsymbol{\nabla}_{0} \boldsymbol{v} \boldsymbol{F}^{-1} \nabla_{0} \Delta \boldsymbol{u} \boldsymbol{F}^{-1}\right) \overline{\boldsymbol{F}}^{\mathrm{T}} \overline{\boldsymbol{F}}
$$$$
+\frac{2}{3} \theta\left[J \operatorname{tr}\left(\boldsymbol{\nabla}_{0} \boldsymbol{v} \boldsymbol{F}^{-1}\right)\right] \theta\left[J \operatorname{tr}\left(\boldsymbol{\nabla}_{0} \Delta \boldsymbol{u} \boldsymbol{F}^{-1}\right)\right] \overline{\boldsymbol{F}}^{\mathrm{T}} \overline{\boldsymbol{F}}
$$$$
+2 \alpha \theta\left[J \operatorname{tr}\left(\boldsymbol{\nabla}_{0} \boldsymbol{v} \boldsymbol{F}^{-1}\right)\right]\left(\overline{\boldsymbol{F}}^{\mathrm{T}} \boldsymbol{\nabla}_{0} \Delta \boldsymbol{u}\right)_{\mathrm{sym}} .
$$ 
One more directional derivative is needed to complete the derivation. To this end, (A.5) and (A.7) are considered in the derivation of

$$
\begin{aligned}
\mathrm{D}\left(\alpha\left(\overline{\boldsymbol{F}}^{\mathrm{T}} \boldsymbol{\nabla}_{0} \boldsymbol{v}\right)_{\mathrm{sym}}\right)[\Delta \boldsymbol{u}]= & +\frac{1}{3 \alpha^{2} J}\left(\overline{\boldsymbol{F}}^{\mathrm{T}} \boldsymbol{\nabla}_{0} \boldsymbol{v}\right)_{\mathrm{sym}} \pi\left[J \operatorname{tr}\left(\boldsymbol{\nabla}_{0} \Delta \boldsymbol{u} \boldsymbol{F}^{-1}\right)\right] \\
& -\frac{1}{3} \alpha\left(\overline{\boldsymbol{F}}^{\mathrm{T}} \boldsymbol{\nabla}_{0} \boldsymbol{v}\right)_{\mathrm{sym}} \operatorname{tr}\left(\boldsymbol{\nabla}_{0} \Delta \boldsymbol{u} \boldsymbol{F}^{-1}\right) \\
& +\frac{1}{3} \alpha\left(\overline{\boldsymbol{F}}^{\mathrm{T}} \boldsymbol{\nabla}_{0} \boldsymbol{v}\right)_{\mathrm{sym}} \theta\left[J \operatorname{tr}\left(\boldsymbol{\nabla}_{0} \Delta \boldsymbol{u} \boldsymbol{F}^{-1}\right)\right] \\
& +\alpha^{2}\left(\left(\boldsymbol{\nabla}_{0} \Delta \boldsymbol{u}\right)^{\mathrm{T}} \boldsymbol{\nabla}_{0} \boldsymbol{v}\right)_{\mathrm{sym}}
\end{aligned}
$$

The last step is to take the second variation of (35), or equivalently, the directional derivative of (41). The derivation is split into a material and a geometric part and motion-independent external forces are assumed. This leads to

$$
\begin{aligned}
\left(\mathrm{D}^{2} \bar{\Pi}(\chi)[\boldsymbol{v}, \Delta \boldsymbol{u}]\right)_{\mathrm{mat}}= & +\frac{1}{3} \int_{\Omega} \theta\left[J \operatorname{tr}\left(\boldsymbol{\nabla}_{0} \boldsymbol{v} \boldsymbol{F}^{-1}\right)\right]\left(\overline{\boldsymbol{F}}^{\mathrm{T}} \overline{\boldsymbol{F}}\right): \mathrm{D} \overline{\boldsymbol{S}}(\chi)[\Delta \boldsymbol{u}] d V \\
& +\int_{\Omega} \alpha\left(\overline{\boldsymbol{F}}^{\mathrm{T}} \boldsymbol{\nabla}_{0} \boldsymbol{v}\right)_{\mathrm{sym}}: \mathrm{D} \overline{\boldsymbol{S}}(\chi)[\Delta \boldsymbol{u}] d V \\
\left(\mathrm{D}^{2} \bar{\Pi}(\chi)[\boldsymbol{v}, \Delta \boldsymbol{u}]\right)_{\mathrm{geo}}= & +\frac{1}{3} \int_{\Omega} \overline{\boldsymbol{S}}: \mathrm{D}\left(\theta\left[J \operatorname{tr}\left(\boldsymbol{\nabla}_{0} \boldsymbol{v} \boldsymbol{F}^{-1}\right)\right] \overline{\boldsymbol{F}}^{\mathrm{T}} \overline{\boldsymbol{F}}\right)[\Delta \boldsymbol{u}] d V \\
& +\int_{\Omega} \overline{\boldsymbol{S}}: \mathrm{D}\left(\alpha\left(\overline{\boldsymbol{F}}^{\mathrm{T}} \boldsymbol{\nabla}_{0} \boldsymbol{v}\right)_{\mathrm{sym}}\right)[\Delta \boldsymbol{u}] d V .
\end{aligned}
$$

Finally, on substituting (B.1) into (B.8) yields the material part of the second variation that was presented in (43b):

$$
\begin{aligned}
\left(\mathrm{D}^{2} \bar{\Pi}(\chi)[\boldsymbol{v}, \Delta \boldsymbol{u}]\right)_{\mathrm{mat}}= & +\frac{1}{9} \int_{\Omega} \theta\left[J \operatorname{tr}\left(\boldsymbol{\nabla}_{0} \boldsymbol{v} \boldsymbol{F}^{-1}\right)\right] \theta\left[J \operatorname{tr}\left(\boldsymbol{\nabla}_{0} \Delta \boldsymbol{u} \boldsymbol{F}^{-1}\right)\right]\left(\overline{\boldsymbol{F}}^{\mathrm{T}} \overline{\boldsymbol{F}}\right): \overline{\mathcal{D}}:\left(\overline{\boldsymbol{F}}^{\mathrm{T}} \overline{\boldsymbol{F}}\right) d V \\
& +\frac{1}{3} \int_{\Omega} \alpha \theta\left[J \operatorname{tr}\left(\boldsymbol{\nabla}_{0} \boldsymbol{v} \boldsymbol{F}^{-1}\right)\right]\left(\overline{\boldsymbol{F}}^{\mathrm{T}} \overline{\boldsymbol{F}}\right): \overline{\mathcal{D}}:\left(\overline{\boldsymbol{F}}^{\mathrm{T}} \boldsymbol{\nabla}_{0} \Delta \boldsymbol{u}\right)_{\mathrm{sym}} d V \\
& +\frac{1}{3} \int_{\Omega} \alpha\left(\overline{\boldsymbol{F}}^{\mathrm{T}} \boldsymbol{\nabla}_{0} \boldsymbol{v}\right)_{\mathrm{sym}}: \overline{\mathcal{D}}:\left(\overline{\boldsymbol{F}}^{\mathrm{T}} \overline{\boldsymbol{F}}\right) \theta\left[J \operatorname{tr}\left(\boldsymbol{\nabla}_{0} \Delta \boldsymbol{u} \boldsymbol{F}^{-1}\right)\right] d V \\
& +\int_{\Omega} \alpha^{2}\left(\overline{\boldsymbol{F}}^{\mathrm{T}} \boldsymbol{\nabla}_{0} \boldsymbol{v}\right)_{\mathrm{sym}}: \overline{\mathcal{D}}:\left(\overline{\boldsymbol{F}}^{\mathrm{T}} \boldsymbol{\nabla}_{0} \Delta \boldsymbol{u}\right)_{\mathrm{sym}} d V
\end{aligned}
$$


and on substituting (B.6) and (B.7) into (B.9) leads to the corresponding geometric part that was presented in $(43 \mathrm{c})$ :

$$
\begin{aligned}
& \left(\mathrm{D}^{2} \bar{\Pi}(\chi)[\boldsymbol{v}, \Delta \boldsymbol{u}]\right)_{\text {geo }}=-\frac{1}{3} \int_{\Omega} \frac{1}{\bar{J}^{2}} \pi\left[J \operatorname{tr}\left(\boldsymbol{\nabla}_{0} \boldsymbol{v} \boldsymbol{F}^{-1}\right)\right] \pi\left[J \operatorname{tr}\left(\boldsymbol{\nabla}_{0} \Delta \boldsymbol{u} \boldsymbol{F}^{-1}\right)\right] \overline{\boldsymbol{S}}:\left(\overline{\boldsymbol{F}}^{\mathrm{T}} \overline{\boldsymbol{F}}\right) d V \\
& +\frac{1}{3} \int_{\Omega} \frac{1}{\bar{J}} \pi\left[J \operatorname{tr}\left(\boldsymbol{\nabla}_{0} \boldsymbol{v} \boldsymbol{F}^{-1}\right) \operatorname{tr}\left(\boldsymbol{\nabla}_{0} \Delta \boldsymbol{u} \boldsymbol{F}^{-1}\right)\right] \overline{\boldsymbol{S}}:\left(\overline{\boldsymbol{F}}^{\mathrm{T}} \overline{\boldsymbol{F}}\right) d V \\
& -\frac{1}{3} \int_{\Omega} \frac{1}{\bar{J}} \pi\left[J \operatorname{tr}\left(\boldsymbol{\nabla}_{0} \boldsymbol{v} \boldsymbol{F}^{-1} \nabla_{0} \Delta \boldsymbol{u} \boldsymbol{F}^{-1}\right)\right] \overline{\boldsymbol{S}}:\left(\overline{\boldsymbol{F}}^{\mathrm{T}} \overline{\boldsymbol{F}}\right) d V \\
& +\frac{1}{3} \int_{\Omega} \operatorname{tr}\left(\boldsymbol{\nabla}_{0} \boldsymbol{v} \boldsymbol{F}^{-1} \nabla_{0} \Delta \boldsymbol{u} \boldsymbol{F}^{-1}\right) \overline{\boldsymbol{S}}:\left(\overline{\boldsymbol{F}}^{\mathrm{T}} \overline{\boldsymbol{F}}\right) d V \\
& +\frac{2}{9} \int_{\Omega} \theta\left[J \operatorname{tr}\left(\boldsymbol{\nabla}_{0} \boldsymbol{v} \boldsymbol{F}^{-1}\right)\right] \theta\left[J \operatorname{tr}\left(\nabla_{0} \Delta \boldsymbol{u} \boldsymbol{F}^{-1}\right)\right] \overline{\boldsymbol{S}}:\left(\overline{\boldsymbol{F}}^{\mathrm{T}} \overline{\boldsymbol{F}}\right) d V \\
& +\frac{2}{3} \int_{\Omega} \alpha \theta\left[J \operatorname{tr}\left(\boldsymbol{\nabla}_{0} \boldsymbol{v} \boldsymbol{F}^{-1}\right)\right] \overline{\boldsymbol{S}}:\left(\overline{\boldsymbol{F}}^{\mathrm{T}} \boldsymbol{\nabla}_{0} \Delta \boldsymbol{u}\right)_{\mathrm{sym}} d V \\
& +\frac{2}{3} \int_{\Omega} \alpha\left(\overline{\boldsymbol{F}}^{\mathrm{T}} \boldsymbol{\nabla}_{0} \boldsymbol{v}\right)_{\mathrm{sym}}: \overline{\boldsymbol{S}} \theta\left[J \operatorname{tr}\left(\boldsymbol{\nabla}_{0} \Delta \boldsymbol{u} \boldsymbol{F}^{-1}\right)\right] d V \\
& +\int_{\Omega} \alpha^{2} \overline{\boldsymbol{S}}:\left[\left(\boldsymbol{\nabla}_{0} \boldsymbol{v}\right)^{\mathrm{T}} \boldsymbol{\nabla}_{0} \Delta \boldsymbol{u}\right]_{\mathrm{sym}} d V .
\end{aligned}
$$

\section{Acknowledgement}

AOB acknowledges the research support of the Chilean National Fund for Scientific and Technological Development (Fondecyt) through grant No. 11110389. NS gratefully acknowledges the research support of the U.S. National Science Foundation through contract grant CMMI-1334783 to the University of California at Davis.

\section{References}

[1] D. N. Arnold, F. Brezzi, M. Fortin, A stable finite element for the Stokes equations, Calcolo 21 (4) (1984) 337-344.

[2] I. Babuška, The finite element method with Lagrangian multipliers, Numerische Mathematik 20 (3) (1973) 179-192. 
[3] F. Brezzi, On the existence, uniqueness and approximation of saddle-point problems arising from Lagrangian multipliers, RAIRO, Analyse Numérique 8 (1974) $129-151$.

[4] O. A. Ladyzhenskaya, The Mathematical Theory of Viscous Incompressible Flows, Gordon and Breach, London, 1969.

[5] F. Auricchio, L. Beirao da Veiga, C. Lovadina, A. Reali, The importance of the exact satisfaction of the incompressibility constraint in nonlinear elasticity: mixed FEMs versus NURBS-based approximations, Computer Methods in Applied Mechanics and Engineering 199 (5-8) (2010) 314-323.

[6] F. Auricchio, L. Beirao da Veiga, C. Lovadina, A. Reali, An analysis of some mixed-enhanced finite element for plane linear elasticity, Computer Methods in Applied Mechanics and Engineering 194 (27-29) (2005) 2947-2968.

[7] C. Lovadina, F. Auricchio, On the enhanced strain technique for elasticity problems, Computers and Structures 81 (8-11) (2003) 777-787.

[8] R. L. Taylor, A mixed-enhanced formulation for tetrahedral finite elements, International Journal for Numerical Methods in Engineering 47 (1-3) (2000) $205-227$.

[9] E. Oñate, J. Rojek, R. L. Taylor, O. C. Zienkiewicz, Finite calculus formulation for incompressible solids using linear triangles and tetrahedra, International Journal for Numerical Methods in Engineering 59 (11) (2004) 1473-1500.

[10] M. Cervera, M. Chiumenti, Q. Valverde, C. Agelet de Saracibar, Mixed linear/linear simplicial elements for incompressible elasticity and plasticity, Com- 
puter Methods in Applied Mechanics and Engineering 192 (49-50) (2003) 52495263.

[11] O. C. Zienkiewicz, J. Rojek, R. L. Taylor, M. Pastor, Triangles and tetrahedra in explicit dynamic codes for solids, International Journal for Numerical Methods in Engineering 43 (3) (1998) 565-583.

[12] E. A. de Souza Neto, F. M. A. Pires, D. R. J. Owen, F-bar-based linear triangles and tetrahedra for finite strain analysis of nearly incompressible solids. Part I: formulation and benchmarking, International Journal for Numerical Methods in Engineering 62 (3) (2005) 353-383.

[13] P. Thoutireddy, J. F. Molinari, E. A. Repetto, M. Ortiz, Tetrahedral composite finite elements, International Journal for Numerical Methods in Engineering 53 (6) (2002) 1337-1351.

[14] Y. Guo, M. Ortiz, T. Belytschko, E. A. Repetto, Triangular composite finite elements, International Journal for Numerical Methods in Engineering 47 (1-3) (2000) 287-316.

[15] J. Bonet, A. J. Burton, A simple average nodal pressure tetrahedral element for incompressible and nearly incompressible dynamic explicit applications, Communications in Numerical Methods in Engineering 14 (5) (1998) 437-449.

[16] C. R. Dohrmann, M. W. Heinstein, J. Jung, S. W. Key, W. R. Witkowski, Node-based uniform strain elements for three-node triangular and four-node tetrahedral meshes, International Journal for Numerical Methods in Engineering 47 (9) (2000) 1549-1568. 
[17] J. Bonet, M. Marriot, O. Hassan, An averaged nodal deformation gradient linear tetrahedral element for large strain explicit dynamic applications, Communications in Numerical Methods in Engineering 17 (8) (2001) 551-561.

[18] M. A. Puso, J. Solberg, A stabilized nodally integrated tetrahedral, International Journal for Numerical Methods in Engineering 67 (6) (2006) 841-867.

[19] P. Krysl, B. Zhu, Locking-free continuum displacement finite elements with nodal integration, International Journal for Numerical Methods in Engineering 76 (7) (2008) 1020-1043.

[20] M. Broccardo, M. Micheloni, P. Krysl, Assumed-deformation gradient finite elements with nodal integration for nearly incompressible large deformation analysis, International Journal for Numerical Methods in Engineering 78 (9) (2009) $1113-1134$.

[21] J. S. Chen, S. Yoon, C. T. Wu, Non-linear version of stabilized conforming nodal integration for Galerkin mesh-free methods, International Journal for Numerical Methods in Engineering 53 (12) (2002) 2587-2615.

[22] M. Puso, E. Zywicz, J. S. Chen, A new stabilized nodal integration approach, in: Meshfree Methods for Partial Differential Equations III, Vol. 57 of Lecture Notes in Computational Science and Engineering, Springer Berlin Heidelberg, 2007, pp. 207-217.

[23] M. A. Puso, J. S. Chen, E. Zywicz, W. Elmer, Meshfree and finite element nodal integration methods, International Journal for Numerical Methods in Engineering 74 (3) (2008) 416-446. 
[24] A. Ortiz-Bernardin, J. S. Hale, C. J. Cyron, Volume-averaged nodal projection method for nearly-incompressible elasticity using meshfree and bubble basis functions, Computer Methods in Applied Mechanics and Engineering 285 (2015) $427-451$.

[25] A. Ortiz, M. A. Puso, N. Sukumar, Maximum-entropy meshfree method for compressible and near-incompressible elasticity, Computer Methods in Applied Mechanics and Engineering 199 (25-28) (2010) 1859-1871.

[26] E. A. de Souza Neto, F. M. Andrade Pires, D. R. J. Owen, F-bar-based linear triangles and tetrahedra for finite strain analysis of nearly incompressible solids. Part I: formulation and benchmarking, International Journal for Numerical Methods in Engineering 62 (3) (2005) 353-383.

[27] T. Elguedj, Y. Bazilevs, V. Calo, T. J. R. Hughes, $\bar{B}$-bar and $\bar{F}$-bar projection methods for nearly incompressible linear and non-linear elasticity and plasticity using higher-order NURBS elements, Computer Methods in Applied Mechanics and Engineering 1 (33-40) (2008) 2667-3172.

[28] C. T. Wu, W. Hu, Meshfree-enriched simplex elements with strain smoothing for the finite element analysis of compressible and nearly incompressible solids, Computer Methods in Applied Mechanics and Engineering 200 (45-46) (2011) 2991-3010.

[29] W. Hu, C. T. Wu, M. Koishi, A displacement-based nonlinear finite element formulation using meshfree-enriched triangular elements for the two-dimensional large deformation analysis of elastomers, Finite Elements in Analysis and Design 50 (0) (2012) 161-172. 
[30] C. T. Wu, M. Koishi, Three-dimensional meshfree-enriched finite element formulation for micromechanical hyperelastic modeling of particulate rubber composites, International Journal for Numerical Methods in Engineering 91 (11) (2012) 1137-1157.

[31] J. S. Chen, C. T. Wu, S. Yoon, Y. You, A stabilized conforming nodal integration for Galerkin mesh-free methods, International Journal for Numerical Methods in Engineering 50 (2) (2001) 435-466.

[32] J. Dolbow, T. Belytschko, Numerical integration of Galerkin weak form in meshfree methods, Computational Mechanics 23 (3) (1999) 219-230.

[33] M. Griebel, M. A. Schweitzer, A particle-partition of unity method. Part II: Efficient cover construction and reliable integration, SIAM Journal on Scientific Computing 23 (5) (2002) 1655-1682.

[34] S. De, K. J. Bathe, The method of finite spheres with improved numerical integration, Computers and Structures 79 (22-25) (2001) 2183-2196.

[35] I. Babuška, U. Banerjee, J. E. Osborn, Q. L. Li, Quadrature for meshless methods, International Journal for Numerical Methods in Engineering 76 (9) (2008) 1434-1470.

[36] I. Babuška, U. Banerjee, J. E. Osborn, Q. Zhang, Effect of numerical integration on meshless methods, Computer Methods in Applied Mechanics and Engineering $198(37-40)(2009)$ 2886-2897.

[37] A. Ortiz, M. Puso, N. Sukumar, Maximum-entropy meshfree method for incompressible media problems, Finite Elements in Analysis and Design 47 (6) (2011) $572-585$. 
[38] Q. Duan, X. Li, H. Zhang, T. Belytschko, Second-order accurate derivatives and integration schemes for meshfree methods, International Journal for Numerical Methods in Engineering 92 (4) (2012) 399-424.

[39] J.-S. Chen, M. Hillman, M. Rüter, An arbitrary order variationally consistent integration for Galerkin meshfree methods, International Journal for Numerical Methods in Engineering 95 (5) (2013) 387-418.

[40] Q. Duan, X. Gao, B. Wang, , X. Li, H. Zhang, T. Belytschko, Y. Shao, Consistent element-free Galerkin method, International Journal for Numerical Methods in Engineering 99 (2) (2014) 79-101.

[41] Q. Duan, X. Gao, B. Wang, X. Li, H. Zhang, A four-point integration scheme with quadratic exactness for three-dimensional element-free Galerkin method based on variationally consistent formulation, Computer Methods in Applied Mechanics and Engineering 280 (0) (2014) 84-116.

[42] T. P. Fries, H. G. Matthies, Classification and overview of meshfree methods, Tech. Rep. Informatikbericht-Nr. 2003-03, Institute of Scientific Computing, Technical University Braunschweig, Braunschweig, Germany (2004).

[43] N. Sukumar, Construction of polygonal interpolants: a maximum entropy approach, International Journal for Numerical Methods in Engineering 61 (12) (2004) 2159-2181.

[44] M. Arroyo, M. Ortiz, Local maximum-entropy approximation schemes: a seamless bridge between finite elements and meshfree methods, International Journal for Numerical Methods in Engineering 65 (13) (2006) 2167-2202. 
[45] N. Sukumar, R. W. Wright, Overview and construction of meshfree basis functions: from moving least squares to entropy approximants, International Journal for Numerical Methods in Engineering 70 (2) (2007) 181-205.

[46] F. Greco, N. Sukumar, Derivatives of maximum-entropy basis functions on the boundary: theory and computations, International Journal for Numerical Methods in Engineering 94 (12) (2013) 1123-1149.

[47] J. Bonet, R. D. Wood, Nonlinear Continuum Mechanics for Finite Element Analysis, Cambridge University Press, UK, 2008.

[48] T. J. R. Hughes, The Finite Element Method: Linear Static and Dynamic Finite Element Analysis, Dover Publications, Inc, Mineola, NY, 2000.

[49] R. Flory, Thermodynamic relations for highly elastic materials, Transactions of the Faraday Society 57 (1969) 829-838.

[50] E. A. de Souza Neto, D. Perić, M. Dutko, D. R. J. Owen, Design of simple low order finite elements for large strain analysis of nearly incompressible solids, International Journal of Solids and Structures 33 (20-22) (1996) 3277-3296.

[51] E. Artioli, G. Castellazzi, P. Krysl, Assumed strain nodally integrated hexahedral finite element formulation for elastoplastic applications, International Journal for Numerical Methods in Engineering 99 (11) (2014) 844-866.

[52] GiD v10.0.9, GiD: the personal pre and post processor, http://www.gidhome.com/ (2011).

[53] P. Hauret, E. Kuhl, M. Ortiz, Diamond elements: a finite element/discretemechanics approximation scheme with guaranteed optimal convergence in in- 
compressible elasticity, International Journal for Numerical Methods in Engineering 72 (3) (2007) 253-294.

[54] S. Reese, P. Wriggers, A stabilization technique to avoid hourglassing in finite elasticity, International Journal for Numerical Methods in Engineering 48 (1) (2000) 79-109.

[55] S. Reese, P. Wriggers, B. Reddy, A new locking-free brick element technique for large deformation problems in elasticity, Computers and Structures 75 (3) (2000) $291-304$. 